FEDERAL RESERVE BANK OF SAN FRANCISCO

WORKING PAPER SERIES

\title{
Risk Aversion, Risk Premia, and the Labor Margin with Generalized Recursive Preferences
}

\author{
Eric T. Swanson \\ Federal Reserve Bank of San Francisco
}

September 2013

Working Paper 2012-17

http://www.frbsf.org/publications/economics/papers/2012/wp12-17bk.pdf

The views in this paper are solely the responsibility of the authors and should not be interpreted as reflecting the views of the Federal Reserve Bank of San Francisco or the Board of Governors of the Federal Reserve System. 


\title{
Risk Aversion, Risk Premia, and the Labor Margin with Generalized Recursive Preferences
}

\author{
Eric T. Swanson \\ Federal Reserve Bank of San Francisco \\ eric.swanson@sf.frb.org \\ http://www.ericswanson.org
}

\begin{abstract}
A flexible labor margin allows households to absorb shocks to asset values with changes in hours worked as well as changes in consumption. This ability to absorb shocks along both margins greatly alters the household's attitudes toward risk, as shown by Swanson (2012). The present paper extends that analysis to the case of generalized recursive preferences, as in Epstein and Zin (1989) and Weil (1989), including multiplier preferences, as in Hansen and Sargent (2001). Understanding risk aversion for these preferences is especially important because they are the primary mechanism being used to bring macroeconomic models into closer agreement with asset pricing facts. Measures of risk aversion commonly used in the literature - including traditional, fixed-labor measures and Cobb-Douglas composite-good measures - show no stable relationship to the equity premium in a standard macroeconomic model, while the closed-form expressions derived in this paper match the equity premium closely. Thus, measuring risk aversion correctly - taking into account the household's labor margin-is necessary for risk aversion to correspond to asset prices in the model.
\end{abstract}

JEL Classification: E44, D81, G11

Version 1.7

September 10, 2013

I thank my discussants, S. Boragan Aruoba and Nicolas Petrosky-Nadeau; Martin Andreasen, Susanto Basu, Rhys Bidder, Ian Dew-Becker, Jesús Fernández-Villaverde, Ivan Jaccard, Dirk Krueger, Elmar Mertens, Adriano Rampini; and seminar participants at the Macro-Finance Society Workshop at Ohio State and at presentations of an earlier paper, "Risk Aversion and the Labor Margin in Dynamic Equilibrium Models," for helpful discussions, comments, and suggestions. The views expressed in this paper, and all errors and omissions, should be regarded as those solely of the author, and are not necessarily those of the individuals listed above, the management of the Federal Reserve Bank of San Francisco, or any other individual in the Federal Reserve System. 


\section{Introduction}

A number of recent studies focus on bringing standard dynamic macroeconomic models into closer agreement with basic asset pricing facts, such as the equity premium or the long-term bond premium. $^{1}$ In these models —indeed, in any consumption-based asset-pricing model—a crucial parameter is risk aversion, the compensation that households require to hold a risky asset. At the same time, a key feature of standard dynamic macroeconomic models is that households have some ability to vary their labor supply. A fundamental difficulty with this line of research, then, is that much of what is known about risk aversion has been derived under the assumption that household labor is exogenously fixed. For example, Arrow (1964) and Pratt (1965) define absolute and relative risk aversion, $-u^{\prime \prime}(c) / u^{\prime}(c)$ and $-c u^{\prime \prime}(c) / u^{\prime}(c)$, in a static model with a single consumption good. Similarly, Epstein and Zin (1989) and Weil (1989) define risk aversion for generalized recursive preferences in a dynamic model without labor (or, equivalently, in which labor is fixed).

Swanson (2012) considers this problem when households have standard expected utility preferences. The present paper extends that analysis to the case of generalized recursive preferences, as in Epstein and Zin (1989) and Weil (1989), including multiplier preferences, as in Hansen and Sargent (2001) and Strzalecki (2011). These preferences are of central importance to the macrofinance literature because they are the primary mechanism being used to bring macroeconomic models into closer agreement with asset pricing facts. ${ }^{2}$ Moreover, there is no conventional wisdom as to what the formula for risk aversion should be for these preferences, with different authors using different ad hoc generalizations of the traditional, fixed-labor measure. The present paper undertakes a systematic and rigorous analysis of this important question.

Intuitively, a flexible labor margin gives households the ability to absorb shocks to asset values with changes in hours worked as well as changes in consumption. This ability to absorb shocks along either or both margins greatly alters the household's attitudes toward risk. For example, with expected utility and period utility function $u\left(c_{t}, l_{t}\right)=c_{t}^{1-\gamma} /(1-\gamma)-\eta l_{t}$, the quantity $-c u_{11} / u_{1}=\gamma$ is often referred to as the household's coefficient of relative risk aversion,

\footnotetext{
${ }^{1}$ For example, Boldrin, Christiano, and Fisher (2001), Tallarini (2000), Rudebusch and Swanson (2008, 2012), Uhlig (2007), Van Binsbergen et al. (2012), Backus, Routledge, and Zin (2008), Gourio (2012, 2013), Palomino (2012), Andreasen (2012, 2013), Colacito and Croce (2012), Dew-Becker (2012), and Kung (2012) all consider asset pricing in dynamic macroeconomic models with a variable labor margin.

2 The vast majority of studies cited in the previous footnote take this approach, the exceptions being Boldrin et al. (2001), Rudebusch and Swanson (2008), and Palomino (2012). One of the main advantages of generalized recursive preferences is that they allow risk aversion to be modeled independently from the household's other preference parameters, such as the intertemporal elasticity of substitution.
} 
but in fact the household is risk neutral with respect to gambles over asset values or wealth (Swanson, 2012). Intuitively, the household is indifferent at the margin between using labor or consumption to absorb a shock to asset values, and the household in this example is clearly risk neutral with respect to gambles over hours. More generally, when $u\left(c_{t}, l_{t}\right)=c_{t}^{1-\gamma} /(1-$ $\gamma)-\eta l_{t}^{1+\chi} /(1+\chi)$, risk aversion equals $\left(\gamma^{-1}+\chi^{-1}\right)^{-1}$, a combination of the parameters on the household's consumption and labor margins, reflecting the fact that the household absorbs shocks along both margins. The present paper shows how to derive closed-form expressions for risk aversion in dynamic equilibrium models with generalized recursive preferences and arbitrary period utility function $u$, taking into account the effects of the household's variable labor margin.

The present paper also shows that risk premia are unrelated to traditional, fixed-labor measures of risk aversion unless labor is, in fact, fixed. In contrast, the closed-form expressions for risk aversion derived in the present paper match risk premia in a standard (flexible-labor) real business cycle model closely. Thus, measuring risk aversion correctly - taking into account the household's labor margin - is necessary for there to be a stable relationship between risk aversion and asset prices in the model. Since many recent studies have focused on bringing standard macroeconomic models into closer agreement with asset prices, it is surprising that so little attention has been paid to measuring risk aversion in these models. The present paper aims to fill that void.

In addition, the present paper demonstrates problems with applying the Epstein-Zin measure of risk aversion, which assumes labor is fixed, to a Cobb-Douglas aggregate of consumption and leisure, as is sometimes done in the literature. Intuitively, the Cobb-Douglas composite good interpretation is problematic if labor and consumption appear separately elsewhere in the model, such as in the production function. Because consumption and leisure do not form a true composite good in the model, a composite-good measure of risk aversion is not necessarily appropriate, and in fact turns out to be poorly correlated with the equity premium in a standard real business cycle model. Instead, the coefficient of relative risk aversion $R^{c}$ defined in the present paperwhich recognizes the household's flexible labor margin but excludes the value of leisure from total household wealth - is more closely related to the equity premium.

There are a few previous studies that extend the Arrow-Pratt definition beyond the onegood, one-period case. Kihlstrom and Mirman (1974) provide an early example of the difficulties involved. In a static, multiple-good setting, Stiglitz (1969) measures risk aversion using the household's indirect utility function rather than utility itself, essentially a special case of Proposition 1 of the present paper. Constantinides (1990) measures risk aversion in a dynamic endowment 
economy (i.e., with fixed labor) using the household's value function, another special case of Proposition 1. Boldrin, Christiano, and Fisher (1997) apply Constantinides' definition to some very simple endowment economy models for which they can compute closed-form expressions for the value function, and hence risk aversion. Uhlig (2007) points out that when households have generalized recursive preferences, leisure affects asset prices because the value function $V$ appears in the household's stochastic discount factor, and $V$ depends on leisure. The present paper builds on these studies by deriving closed-form solutions for risk aversion in dynamic equilibrium models in general, demonstrating the importance of the labor margin, and showing the tight link between risk aversion, properly defined, and asset prices in these models.

A number of empirical studies also support the view that households vary their labor supply in response to portfolio shocks. For example, Imbens, Rubin, and Sacerdote (2001) show that individuals who win a prize in the lottery reduce their labor supply significantly. Coile and Levine (2009) document that older individuals are less likely to retire after the stock market performs poorly, and Coronado and Perozek (2003) find that households retire earlier when the stock market performs well. More generally, Pencavel (1986) surveys estimates of the wealth effect on labor supply and finds it to be significantly negative.

The remainder of the paper proceeds as follows. Section 2 defines the dynamic equilibrium framework used in the analysis. Section 3 derives closed-form expressions for risk aversion in the model. Section 4 demonstrates the close connection between risk aversion and Lucas-Breeden asset prices in the model, both theoretically and with numerical examples. Section 5 verifies the accuracy of the closed-form expressions for risk aversion using numerical methods. Section 6 extends the results to the case of balanced growth. Section 7 provides the corresponding expressions for the case of multiplier preferences. Section 8 discusses some general implications and concludes. An Appendix provides details of proofs and numerical solutions that are outlined in the main text.

\section{Dynamic Equilibrium Framework}

\subsection{Generalized Recursive Preferences and Value Function}

Time is discrete and continues forever. At each time $t$, the household receives a utility flow $u\left(c_{t}, l_{t}\right)$, where $\left(c_{t}, l_{t}\right) \in \Omega \subseteq \mathbb{R}^{2}$ denotes the household's choice of consumption and hours worked in period $t$. The period utility function $u$ is assumed to satisfy the following regularity conditions: 
Assumption 1. The function $u: \Omega \rightarrow \mathbb{R}$ is increasing in its first argument, decreasing in its second, twice-differentiable, and strictly concave.

The household faces a flow budget constraint in each period,

$$
a_{t+1}=\left(1+r_{t}\right) a_{t}+w_{t} l_{t}+d_{t}-c_{t}
$$

and a no-Ponzi-scheme condition,

$$
\lim _{T \rightarrow \infty} \prod_{\tau=t}^{T}\left(1+r_{\tau+1}\right)^{-1} a_{T+1} \geq 0
$$

where $a_{t}$ denotes beginning-of-period assets and $w_{t}, r_{t}$, and $d_{t}$ denote the real wage, real interest rate, and net transfer payments to the household, respectively. There is a finite-dimensional Markov state vector $\theta_{t}$ that is exogenous to the household and governs the processes for $w_{t}, r_{t}$, and $d_{t}$. Before choosing $\left(c_{t}, l_{t}\right)$ in each period $t$, the household observes $\theta_{t}$ and hence $w_{t}, r_{t}$, and $d_{t}$. The state vector and information set of the household's optimization problem at each date $t$ is thus $\left(a_{t} ; \theta_{t}\right)$. Let $X$ denote the domain of $\left(a_{t} ; \theta_{t}\right)$, and $\Gamma: X \rightarrow \Omega$ describe the set-valued correspondence of feasible choices for $\left(c_{t}, l_{t}\right)$ for each given $\left(a_{t} ; \theta_{t}\right)$.

Let $\left(c^{t}, l^{t}\right) \equiv\left\{\left(c_{\tau}, l_{\tau}\right)\right\}_{\tau=t}^{\infty}$ denote a state-contingent plan for household consumption and labor from time $t$ onward, where the explicit state-dependence of the plan is suppressed to reduce notation. Following Epstein and Zin (1989) and Weil (1989), the household has preferences over state-contingent plans ordered by the recursive functional

$$
\widetilde{V}\left(c^{t}, l^{t}\right)=u\left(c_{t}, l_{t}\right)+\beta\left[E_{t} \widetilde{V}\left(c^{t+1}, l^{t+1}\right)^{1-\alpha}\right]^{1 /(1-\alpha)},
$$

where $\beta \in(0,1), \alpha \in \mathbb{R}, E_{t}$ denotes the mathematical expectation conditional on the household's information set at time $t$, and $\left(c^{t+1}, l^{t+1}\right)$ denotes the state-contingent plan $\left(c^{t}, l^{t}\right)$ from date $t+1$ forward. ${ }^{3}$ Note that equation (3) has the same form as expected utility preferences, but with the expectation operator "twisted" and "untwisted" by the coefficient $1-\alpha$. When $\alpha=0$, (3) reduces to the special case of expected utility. When $\alpha \neq 0$, the intertemporal elasticity of substitution over deterministic consumption paths in (3) is the same as for expected utility, but the household's risk aversion with respect to gambles over future utility flows is amplified (or attenuated) by the additional curvature parameter $\alpha .^{4}$

\footnotetext{
${ }^{3}$ The case of multiplier preferences, as in Hansen and Sargent (2001) and Strzalecki (2011), is considered explicitly in Section 7, below. However, much of the discussion here is also relevant for that case.

${ }^{4}$ The case $\alpha=1$ is understood to correspond to $\widetilde{V}\left(c^{t}, l^{t}\right)=u\left(c_{t}, l_{t}\right)+\beta \exp \left[E_{t} \log \tilde{V}\left(c^{t+1}, l^{t+1}\right)\right]$.
} 
The household's "generalized value function" $V: X \rightarrow \mathbb{R}$ is defined to be the maximized value of (3), subject to the budget constraint (1)-(2). $V$ satisfies the recursive equation

$$
V\left(a_{t} ; \theta_{t}\right)=\max _{\left(c_{t}, l_{t}\right) \in \Gamma\left(a_{t} ; \theta_{t}\right)} u\left(c_{t}, l_{t}\right)+\beta\left(E_{t} V\left(a_{t+1} ; \theta_{t+1}\right)^{1-\alpha}\right)^{1 /(1-\alpha)},
$$

where $a_{t+1}$ is given by (1). Technical conditions for the existence and uniqueness of $V$ are discussed shortly.

Note that many authors use an alternate specification for the generalized value function,

$$
U\left(a_{t} ; \theta_{t}\right)=\max _{\left(c_{t}, l_{t}\right) \in \Gamma\left(a_{t} ; \theta_{t}\right)}\left[\widetilde{u}\left(c_{t}, l_{t}\right)^{\rho}+\beta\left(E_{t} U\left(a_{t+1} ; \theta_{t+1}\right)^{\widetilde{\alpha}}\right)^{\rho / \widetilde{\alpha}}\right]^{1 / \rho},
$$

where $\rho \in \mathbb{R}, \rho<1$. This specification follows Epstein and Zin's (1989) original definition more closely, where those authors take $\widetilde{u}\left(c_{t}, l_{t}\right)=c_{t}$ in their framework without labor. However, setting $V=U^{\rho}, u=\widetilde{u}^{\rho}$, and $\alpha=1-\widetilde{\alpha} / \rho$, this can be seen to correspond exactly to (4). ${ }^{5}$ Moreover, (4) has a much clearer relationship than (5) to standard dynamic programming results, regularity conditions, and first-order conditions: for example, (4) requires concavity of $u$ while (5) requires concavity of $\widetilde{u}^{\rho}$, and the Benveniste-Scheinkman equation for (4) is the usual $V_{1}=\left(1+r_{t}\right) u_{1}$ rather than $U_{1}=\left(1+r_{t}\right) U^{(1-\rho) / \rho} \widetilde{u}^{\rho-1} \widetilde{u}_{1}$. That is, the marginal value of wealth in (4) is just the usual marginal utility of consumption rather than something much more complicated.

A few technical conditions are required to ensure that (3)-(4) are well-defined. First, to avoid complex numbers:

Assumption 2. Either $u: \Omega \rightarrow[0, \infty)$ or $u: \Omega \rightarrow(-\infty, 0]$.

In the latter case, it is natural to take $\widetilde{V} \leq 0, V \leq 0$, and interpret (3) as

$$
\widetilde{V}\left(c^{t}, l^{t}\right)=u\left(c_{t}, l_{t}\right)-\beta\left[E_{t}\left(-\widetilde{V}\left(c^{t+1}, l^{t+1}\right)\right)^{1-\alpha}\right]^{1 /(1-\alpha)},
$$

and similarly for (4). Note that (5) requires this same restriction, for the same reasons. ${ }^{6}$

Technical conditions that ensure the existence and uniqueness of $V$ are tangential to the main points of the present paper, so it is simply assumed that: ${ }^{7}$

\footnotetext{
${ }^{5}$ For the case $\rho<0$, set $V=-U^{\rho}$ and $u=-\widetilde{u}^{\rho}$. The case $\rho=0$ corresponds to multiplier preferences, considered in Section 7.

${ }^{6}$ The assumption that either $u \geq 0$ or $u \leq 0$ is not very restrictive in practice. For example, restrictions can be placed on $\Omega$ or $\Gamma$ and a constant added to $u$ such that $u$ never takes on negative (or positive) values. Alternatively, for local analysis around a steady state, the restriction is satisfied so long as $u \neq 0$ in steady state, since then $u \geq 0$ or $u \leq 0$ holds locally. Note that Assumption 2 is not required for multiplier preferences; see Section 7 .

${ }^{7}$ Stokey and Lucas (1990), Alvarez and Stokey (1998), and Rincón-Zapatero and Rodríguez-Palmero (2003) provide different sets of sufficient conditions that ensure Assumption 3 is satisfied for the case $\alpha=0$. Sufficient conditions for general $\alpha$ have not yet been derived in the literature, but Epstein and Zin (1989) and Marinacci and Montrucchio (2010) provide important results for the fixed-labor case.
} 
Assumption 3. A solution $V: X \rightarrow \mathbb{R}$ to the household's generalized Bellman equation (4) exists and is unique, continuous, and concave.

The same technical conditions, plus Assumption 1, guarantee the existence of a unique optimal choice for $\left(c_{t}, l_{t}\right)$ at each point in time, given $\left(a_{t} ; \theta_{t}\right)$. Let $c_{t}^{*} \equiv c^{*}\left(a_{t} ; \theta_{t}\right)$ and $l_{t}^{*} \equiv l^{*}\left(a_{t} ; \theta_{t}\right)$ denote the household's optimal choices of $c_{t}$ and $l_{t}$ as functions of the state $\left(a_{t} ; \theta_{t}\right)$. Then $V$ can be written as

$$
V\left(a_{t} ; \theta_{t}\right)=u\left(c_{t}^{*}, l_{t}^{*}\right)+\beta\left(E_{t} V\left(a_{t+1}^{*} ; \theta_{t+1}\right)^{1-\alpha}\right)^{1 /(1-\alpha)},
$$

where $a_{t+1}^{*} \equiv\left(1+r_{t}\right) a_{t}+w_{t} l_{t}^{*}+d_{t}-c_{t}^{*}$. These solutions are also assumed to be interior:

Assumption 4. For any $\left(a_{t} ; \theta_{t}\right) \in X$, the household's optimal choice $\left(c_{t}^{*}, l_{t}^{*}\right)$ exists, is unique, and lies in the interior of $\Gamma\left(a_{t} ; \theta_{t}\right)$.

Intuitively, Assumption 4 requires the partial derivatives of $u$ to grow sufficiently large toward the boundary that only interior solutions for $c_{t}^{*}$ and $l_{t}^{*}$ are optimal for any $\left(a_{t} ; \theta_{t}\right) \in X$.

Assumptions 1-4 guarantee that $V$ is continuously differentiable with respect to $a$ and satisfies the Benveniste-Scheinkman equation, but slightly more than this will be required below:

Assumption 5. For any $\left(a_{t} ; \theta_{t}\right)$ in the interior of $X$, the second derivative of $V$ with respect to its first argument, $V_{11}\left(a_{t} ; \theta_{t}\right)$, exists.

Assumption 5 also implies differentiability of the optimal policy functions, $c^{*}$ and $l^{*}$, with respect to $a_{t}$. Santos (1991) provides relatively mild sufficient conditions for this assumption to be satisfied when $\alpha=0$; intuitively, $u$ must be strongly concave.

\subsection{Representative Household and Steady State Assumptions}

Up to this point, the analysis has focused on a single household in isolation, leaving the other households of the model and the production side of the economy unspecified. Implicitly, the other households and production sector jointly determine the process for $\theta_{t}$ (and hence $w_{t}, r_{t}$, and $d_{t}$ ), and much of the analysis below does not need to be any more specific about these processes than this. However, to move from general expressions for risk aversion to more concrete, closed-form expressions, three standard assumptions from the macroeconomics literature are adopted: ${ }^{8}$

Assumption 6. The household is infinitesimal.

Assumption 7. The household is representative.

\footnotetext{
${ }^{8}$ Alternative assumptions about the nature of the other households in the model or the production sector may also allow for closed-form expressions for risk aversion. However, the assumptions used here are the most standard.
} 
Assumption 8. The model has a nonstochastic steady state, at which $x_{t}=x_{t+k}$ for all $k=1,2$, $\ldots$, and $x \in\{c, l, a, w, r, d, \theta\}$.

Assumption 6 implies that an individual household's choices for $c_{t}$ and $l_{t}$ have no effect on the aggregate quantities $w_{t}, r_{t}, d_{t}$, and $\theta_{t}$. Assumption 7 implies that, when the economy is at the nonstochastic steady state, any individual household finds it optimal to choose the steady-state values of $c$ and $l$ given $a$ and $\theta$. Throughout the text, a variable without its time subscript $t$ is used to denote its steady-state value. ${ }^{9}$

It is important to note that Assumptions 7-8 do not prohibit offering an individual household a hypothetical gamble of the type described below. The steady state of the model serves only as a reference point around which the aggregate variables $w, r, d$, and $\theta$ and the other households' choices of $c, l$, and $a$ can be predicted with certainty. This reference point is important because it is there that closed-form expressions for risk aversion can be computed.

Finally, many dynamic models do not have a steady state per se, but rather a balanced growth path, as in King, Plosser, and Rebelo (1988). The results below carry through essentially unchanged to the case of balanced growth. For ease of exposition, Sections 3-5 restrict attention to the case of a steady state, while Section 6 shows the adjustments required under the more general:

Assumption 8'. The model has a balanced growth path that can be renormalized to a nonstochastic steady state after a suitable change of variables.

\section{Risk Aversion}

\subsection{The Coefficient of Absolute Risk Aversion}

The household's attitudes toward risk at time $t$ generally depend on the household's state vector at time $t,\left(a_{t} ; \theta_{t}\right)$. Given this state, the household's aversion to a hypothetical one-shot gamble in period $t$ of the form

$$
a_{t+1}=\left(1+r_{t}\right) a_{t}+w_{t} l_{t}+d_{t}-c_{t}+\sigma \varepsilon_{t+1}
$$

can be considered, where $\varepsilon_{t+1}$ is a random variable representing the gamble, with bounded support $[\underline{\varepsilon}, \bar{\varepsilon}]$, mean zero, unit variance, independent of $\theta_{\tau}$ for all times $\tau$, and independent of $a_{\tau}, c_{\tau}$, and $l_{\tau}$

\footnotetext{
${ }^{9}$ Let the exogenous state $\theta_{t}$ contain the variances of any shocks to the model, so that $(a ; \theta)$ denotes the nonstochastic steady state, with the variances of any shocks (other than the hypothetical gamble described in the next section) set equal to zero; $c(a ; \theta)$ corresponds to the household's optimal consumption choice at the nonstochastic steady state, etc.
} 
for all $\tau \leq t$. A few words about (7) are in order: First, the gamble is dated $t+1$ to clarify that its outcome is not in the household's information set at time $t$. Second, $c_{t}$ cannot be made the subject of the gamble without substantial modifications to the household's optimization problem, because $c_{t}$ is a choice variable under control of the household at time $t$. However, (7) is clearly equivalent to a one-shot gamble over net transfers $d_{t}$ or asset returns $r_{t}$, both of which are exogenous to the household. ${ }^{10}$ Indeed, thinking of the gamble as being over $r_{t}$ helps to illuminate the connection between (7) and the price of risky assets, which will be discussed further in Section 4. As shown there, the household's aversion to the gamble in (7) is directly linked to the premium households require to hold risky assets.

Following Arrow (1964) and Pratt (1965), one can ask what one-time fee $\mu$ the household would be willing to pay in period $t$ to avoid the gamble in (7):

$$
a_{t+1}=\left(1+r_{t}\right) a_{t}+w_{t} l_{t}+d_{t}-c_{t}-\mu \text {. }
$$

The quantity $\mu$ that makes the household just indifferent between (7) and (8), for infinitesimal $\sigma$ and $\mu$, is the household's coefficient of absolute risk aversion. Formally, this corresponds to the following definition:

Definition 1. Let $\left(a_{t} ; \theta_{t}\right)$ be an interior point of $X$. Let $\hat{V}\left(a_{t} ; \theta_{t} ; \sigma\right)$ denote the value function for the household's optimization problem inclusive of the one-shot gamble $(7)$, and let $\mu\left(a_{t} ; \theta_{t} ; \sigma\right)$ denote the value of $\mu$ that satisfies $V\left(a_{t}-\frac{\mu}{1+r_{t}} ; \theta_{t}\right)=\hat{V}\left(a_{t} ; \theta_{t} ; \sigma\right)$. The household's coefficient of absolute risk aversion at $\left(a_{t} ; \theta_{t}\right)$, denoted $R^{a}\left(a_{t} ; \theta_{t}\right)$, is given by $R^{a}\left(a_{t} ; \theta_{t}\right)=\lim _{\sigma \rightarrow 0} \mu\left(a_{t} ; \theta_{t} ; \sigma\right) /\left(\sigma^{2} / 2\right)$.

In Definition 1, $\mu\left(a_{t} ; \theta_{t} ; \sigma\right)$ denotes the household's "willingness to pay" to avoid a one-shot gamble of size $\sigma$ in (7). As in Arrow (1964) and Pratt (1965), $R^{a}$ denotes the limit of the household's willingness to pay per unit of variance as this variance becomes small. Note that $R^{a}\left(a_{t} ; \theta_{t}\right)$ depends on the economic state because $\mu\left(a_{t} ; \theta_{t} ; \sigma\right)$ depends on that state. Proposition 1 shows that $\hat{V}\left(a_{t} ; \theta_{t} ; \sigma\right), \mu\left(a_{t} ; \theta_{t} ; \sigma\right)$, and $R^{a}\left(a_{t} ; \theta_{t}\right)$ in Definition 1 are well-defined and derives the expression for $R^{a}\left(a_{t} ; \theta_{t}\right)$ :

Proposition 1. Let $\left(a_{t} ; \theta_{t}\right)$ be an interior point of $X$. Given Assumptions $1-6, \hat{V}\left(a_{t} ; \theta_{t} ; \sigma\right)$, $\mu\left(a_{t} ; \theta_{t} ; \sigma\right)$, and $R^{a}\left(a_{t} ; \theta_{t}\right)$ exist and

$$
R^{a}\left(a_{t} ; \theta_{t}\right)=\frac{-E_{t}\left[V\left(a_{t+1}^{*} ; \theta_{t+1}\right)^{-\alpha} V_{11}\left(a_{t+1}^{*} ; \theta_{t+1}\right)-\alpha V\left(a_{t+1}^{*} ; \theta_{t+1}\right)^{-\alpha-1} V_{1}\left(a_{t+1}^{*} ; \theta_{t+1}\right)^{2}\right]}{E_{t} V\left(a_{t+1}^{*} ; \theta_{t+1}\right)^{-\alpha} V_{1}\left(a_{t+1}^{*} ; \theta_{t+1}\right)}
$$

\footnotetext{
${ }^{10}$ In this case, the realized transfer $d_{t}+\sigma \varepsilon_{t+1}$, or asset return $r_{t}+\sigma \varepsilon_{t+1}$, would not be in the household's time- $t$ information set, $\left(a_{t} ; \theta_{t}\right)$.
} 
where $V_{1}$ and $V_{11}$ denote the first and second partial derivatives of $V$ with respect to its first argument. Given Assumptions 7-8, equation (9) can be evaluated at the steady state to yield:

$$
R^{a}(a ; \theta)=\frac{-V_{11}(a ; \theta)}{V_{1}(a ; \theta)}+\alpha \frac{V_{1}(a ; \theta)}{V(a ; \theta)}
$$

Proof: See Appendix. ${ }^{11}$

Note that risk aversion in the dynamic case is related to the curvature of the value function with respect to wealth rather than the curvature of $u$ with respect to consumption. When $\alpha=0$, (10) reduces to $-V_{11} / V_{1}$, the standard "folk wisdom" value for risk aversion in the dynamic expected utility framework. ${ }^{12}$ For general $\alpha$, there is no folk wisdom for what the formula for risk aversion should be, highlighting the importance of the present paper's methods and results.

Risk aversion in (10) can be decomposed into the sum of two components: the first term on the right-hand side is essentially intratemporal and holds no matter what the value of $\alpha$, while the second term captures the household's additional aversion to risky utility flows in the future and is closely related to $\alpha$. For $u, V \geq 0$, larger values of $\alpha$ imply higher levels of risk aversion. ${ }^{13}$ For $u, V \leq 0$, the opposite is true: larger values of $\alpha$ imply lower levels of risk aversion.

A practical difficulty with Proposition 1 is that closed-form expressions for $V$ do not exist in general, even for the simplest dynamic models with labor. One can solve this problem by observing that $V_{1}$ and $V_{11}$ often can be computed even when closed-form solutions for $V$ cannot be. For example, the Benveniste-Scheinkman equation,

$$
V_{1}\left(a_{t} ; \theta_{t}\right)=\left(1+r_{t}\right) u_{1}\left(c_{t}^{*}, l_{t}^{*}\right)
$$

states that the marginal value of a dollar of assets equals the marginal utility of consumption times $1+r_{t}$ (the interest rate appears here because beginning-of-period assets in the model generate

${ }^{11}$ When generalized recursive preferences are written in the form (5), the corresponding expressions are

$$
R^{a}\left(a_{t} ; \theta_{t}\right)=\frac{-E_{t}\left[U\left(a_{t+1}^{*} ; \theta_{t+1}\right)^{\widetilde{\alpha}-1} U_{11}\left(a_{t+1}^{*} ; \theta_{t+1}\right)+(\widetilde{\alpha}-1) U\left(a_{t+1}^{*} ; \theta_{t+1}\right)^{\widetilde{\alpha}-2} U_{1}\left(a_{t+1}^{*} ; \theta_{t+1}\right)^{2}\right]}{E_{t} U\left(a_{t+1}^{*} ; \theta_{t+1}\right)^{\widetilde{\alpha}-1} U_{1}\left(a_{t+1}^{*} ; \theta_{t+1}\right)}
$$

and

$$
R^{a}(a ; \theta)=\frac{-U_{11}(a ; \theta)}{U_{1}(a ; \theta)}+(1-\widetilde{\alpha}) \frac{U_{1}(a ; \theta)}{U(a ; \theta)}
$$

12 See, e.g., Constantinides (1990), Farmer (1990), Campbell and Cochrane (1999), and Flavin and Nakagawa (2008). Swanson (2012) derives this folk wisdom value rigorously using the same methods as the present paper.

13 Sufficiently low or negative values of $\alpha$ can imply risk-loving behavior, $R^{a}(a ; \theta)<0$. The parameter $\alpha$ also determines the household's preference for early vs. late resolution of uncertainty, as discussed in Kreps and Porteus (1978) and Epstein and Zin (1989), because $\alpha$ determines the household's aversion to uncertainty about future utility flows $V$. For $u, V \geq 0$, the household prefers early resolution of uncertainty if and only if $\alpha>0$; for $u, V \leq 0$, the household prefers early resolution if and only if $\alpha<0$. These conditions correspond to the criterion $\widetilde{\alpha}<\rho$ in (5), emphasized by Epstein and Zin (1989). 
income in period $t) .{ }^{14} \operatorname{In}(11), u_{1}$ is a known function. Although closed-form solutions for the functions $c^{*}$ and $l^{*}$ are not known in general, the points $c_{t}^{*}$ and $l_{t}^{*}$ often are known-for example, when they are evaluated at the nonstochastic steady state, $c$ and $l$. Thus, one can compute $V_{1}$ at the nonstochastic steady state by evaluating (11) at that point.

The second derivative $V_{11}$ can be computed by noting that (11) holds for general $a_{t}$; hence it can be differentiated to yield:

$$
V_{11}\left(a_{t} ; \theta_{t}\right)=\left(1+r_{t}\right)\left[u_{11}\left(c_{t}^{*}, l_{t}^{*}\right) \frac{\partial c_{t}^{*}}{\partial a_{t}}+u_{12}\left(c_{t}^{*}, l_{t}^{*}\right) \frac{\partial l_{t}^{*}}{\partial a_{t}}\right]
$$

All that remains is to find the derivatives $\partial c_{t}^{*} / \partial a_{t}$ and $\partial l_{t}^{*} / \partial a_{t}$.

One can solve for $\partial l_{t}^{*} / \partial a_{t}$ by differentiating the household's intratemporal optimality condition,

$$
-u_{2}\left(c_{t}^{*}, l_{t}^{*}\right)=w_{t} u_{1}\left(c_{t}^{*}, l_{t}^{*}\right)
$$

with respect to $a_{t}$, and rearranging terms to yield:

$$
\frac{\partial l_{t}^{*}}{\partial a_{t}}=-\lambda_{t} \frac{\partial c_{t}^{*}}{\partial a_{t}}
$$

where

$$
\lambda_{t} \equiv \frac{w_{t} u_{11}\left(c_{t}^{*}, l_{t}^{*}\right)+u_{12}\left(c_{t}^{*}, l_{t}^{*}\right)}{u_{22}\left(c_{t}^{*}, l_{t}^{*}\right)+w_{t} u_{12}\left(c_{t}^{*}, l_{t}^{*}\right)}=\frac{u_{1}\left(c_{t}^{*}, l_{t}^{*}\right) u_{12}\left(c_{t}^{*}, l_{t}^{*}\right)-u_{2}\left(c_{t}^{*}, l_{t}^{*}\right) u_{11}\left(c_{t}^{*}, l_{t}^{*}\right)}{u_{1}\left(c_{t}^{*}, l_{t}^{*}\right) u_{22}\left(c_{t}^{*}, l_{t}^{*}\right)-u_{2}\left(c_{t}^{*}, l_{t}^{*}\right) u_{12}\left(c_{t}^{*}, l_{t}^{*}\right)} .
$$

Note that, if consumption and leisure in period $t$ are normal goods, then $\lambda_{t}>0$, although this restriction is not required below. It now only remains to solve for the derivative $\partial c_{t}^{*} / \partial a_{t}$.

Intuitively, $\partial c_{t}^{*} / \partial a_{t}$ should not be too difficult to compute: it is just the household's marginal propensity to consume today out of a change in assets, which can be deduced from the household's Euler equation and budget constraint: ${ }^{15}$

Lemma 2. Given Assumptions 1-8, the household's marginal propensity to consume out of wealth in a neighborhood of the nonstochastic steady state satisfies

$$
\frac{\partial c_{t}^{*}}{\partial a_{t}}=E_{t} \frac{\partial c_{t+1}^{*}}{\partial a_{t}}=E_{t} \frac{\partial c_{t+k}^{*}}{\partial a_{t}}, \quad k=1,2,3, \ldots
$$

and

$$
\frac{\partial c_{t}^{*}}{\partial a_{t}}=\frac{r}{1+w \lambda}
$$

\footnotetext{
${ }^{14}$ The Benveniste-Scheinkman equation (11) holds for generalized recursive preferences as well as expected utility. See the proof of Proposition 1 in the Appendix.

${ }^{15}$ The notation $\frac{\partial c_{t+1}^{*}}{\partial a_{t}}$ is taken to mean $\frac{\partial c_{t+1}^{*}}{\partial a_{t+1}} \frac{d a_{t+1}^{*}}{d a_{t}}=\frac{\partial c_{t+1}^{*}}{\partial a_{t+1}}\left[1+r_{t+1}+w_{t} \frac{\partial l_{t}^{*}}{\partial a_{t}}-\frac{\partial c_{t}^{*}}{\partial a_{t}}\right]$, and analogously for $\frac{\partial c_{t+2}^{*}}{\partial a_{t}}, \frac{\partial c_{t+3}^{*}}{\partial a_{t}}$, etc.
} 
Proof: See Appendix.

In other words, starting near the nonstochastic steady state, the household's optimal change in consumption today in response to an increase in assets must be the same as the expected change in consumption tomorrow, and the expected change in consumption at each future date $t+k$. Note that this equality does not follow from the steady-state assumption-for example, in a model with internal habits, the individual household's optimal consumption response to a change in assets increases gradually over time, even starting from steady state.

According to Lemma 2, the household's optimal response to a unit increase in assets is to raise consumption in every period by the extra asset income, $r$ (the "golden rule"), adjusted downward by the amount $1+w \lambda$, which takes into account the household's decrease in hours worked and labor income. Thus, Lemma 2 represents a "modified golden rule" that takes into account the household's labor margin.

The household's coefficient of absolute risk aversion can now be computed. Substituting (11), (12), (14), and (17) into (10) proves the following:

Proposition 3. Given Assumptions 1-8, the household's coefficient of absolute risk aversion, $R^{a}\left(a_{t} ; \theta_{t}\right)$, evaluated at steady state, satisfies

$$
R^{a}(a ; \theta)=\frac{-u_{11}+\lambda u_{12}}{u_{1}} \frac{r}{1+w \lambda}+\alpha \frac{r u_{1}}{u}
$$

where $u_{1}, u_{11}$, and $u_{12}$ denote the corresponding partial derivatives of $u$ evaluated at the steady state $(c, l)$, and $\lambda$ is given by (15) evaluated at steady state.

There are several features of Proposition 3 worth noting. First, when $\alpha=0$, equation (18) reduces to the expressions derived in Swanson (2012) for the case of expected utility. When $\alpha=0$ and labor is fixed $(\lambda=0)$, risk aversion in (18) reduces further to $-r u_{11} / u_{1}$, which is just the usual Arrow-Pratt definition multiplied by $r$, a scaling factor that translates between assets and current-period consumption units. ${ }^{16}$

When $u \geq 0$ everywhere, risk aversion is increasing in $\alpha$, and when $u \leq 0, R^{a}$ is decreasing in $\alpha$, as observed after Proposition 1. Multiplying $u$ by a constant has no effect on risk aversion, but an additive translation of $u$ does affect risk aversion if $\alpha \neq 0$, because it changes the "twisted" expectation in equation (4). When $\alpha \neq 0$ and labor is fixed $(\lambda=0)$, equation (18) reduces to

\footnotetext{
${ }^{16}$ A gamble over a lump sum of $\$ X$ is equivalent here to a gamble over an annuity of $\$ X / r$. Thus, even though $V_{11} / V_{1}$ is different from $u_{11} / u_{1}$ by a factor of $r$, this difference is exactly the same as a change from lump-sum to annuity units. Thus, the difference in scale is essentially one of units.
} 
$\frac{-u_{11}}{u_{1}}+\alpha \frac{u_{1}}{u}$, times $r$, corresponding to the standard formula for absolute risk aversion in an Epstein-Zin-Weil endowment economy (see Example 1, below). ${ }^{17}$

When $\lambda \neq 0$, households can partially offset shocks to income through changes in hours worked. Even when consumption and labor are additively separable in $u\left(u_{12}=0\right), c_{t}^{*}$ and labor supply are indirectly connected through the household's budget constraint. When $u_{12} \neq 0$, risk aversion is further attenuated or amplified by the direct interaction between consumption and labor in utility, $u_{12}$. Note, however, that regardless of the signs of $\lambda$ and $u_{12}, R^{a}$ is always reduced when households can vary their labor supply:

\section{Corollary 4.}

$$
R^{a}(a ; \theta) \leq \frac{-r u_{11}}{u_{1}}+\alpha \frac{r u_{1}}{u}
$$

Proof: Substituting in for $\lambda$ and $w$, the first term in (18) can be written as:

$$
\frac{-r u_{11}}{u_{1}} \frac{u_{11} u_{22}-u_{12}^{2}}{u_{11} u_{22}-2 \frac{u_{2}}{u_{1}} u_{11} u_{12}+\left(\frac{u_{2}}{u_{1}}\right)^{2} u_{11}^{2}}=\frac{-r u_{11}}{u_{1}} \frac{1}{1+\frac{\left(\frac{u_{2}}{u_{1}} u_{11}-u_{12}\right)^{2}}{u_{11} u_{22}-u_{12}^{2}}} .
$$

Strict concavity of $u$ implies $u_{11} u_{22}-u_{12}^{2}>0$, hence the right-hand side of (20) is less than or equal to $-r u_{11} / u_{1}$.

The right-hand side of (19) is the formula for risk aversion with generalized recursive preferences when labor is exogenously fixed.

The household's labor margin can have dramatic effects on risk aversion. Even if $-u_{11} / u_{1}$ is very large, the first term in $(20)$ can be arbitrarily small as the matrix discriminant, $u_{11} u_{22}-u_{12}^{2}$, approaches zero. In other words, the first term in (20) depends on the concavity of $u$ in all dimensions rather than just in one dimension. The second term in (19)-(20), $\alpha r u_{1} / u$, is not directly affected by a change from a fixed-labor to flexible-labor assumption, however.

Some examples of risk aversion calculations are provided in Section 3.3, below, after first defining relative risk aversion.

\footnotetext{
${ }^{17}$ When generalized recursive preferences are written in the form (5), $w=-\widetilde{u}_{2} / \widetilde{u}_{1}, \lambda=\frac{w \widetilde{u}_{11}+\widetilde{u}_{12}}{\widetilde{u}_{22}+w \widetilde{u}_{12}}$, and $R^{a}(a ; \theta)=\left[\frac{-\widetilde{u}_{11}+\lambda \widetilde{u}_{12}}{\widetilde{u}_{1}}+(\rho-1) \frac{-\widetilde{u}_{1}+\lambda \widetilde{u}_{2}}{\widetilde{u}}\right] \frac{r}{1+w \lambda}+(\rho-\widetilde{\alpha}) \frac{r \widetilde{u}_{1}}{\widetilde{u}}$.
}

This expression is somewhat more complicated than (18), owing to the more complicated derivatives of (5). When $\lambda=0$ and $\widetilde{u}=c$, this reduces to $(1-\widetilde{\alpha}) / c$, the traditional fixed-labor measure of absolute risk aversion in Epstein and Zin (1989), Weil (1989), and Example 1. 


\subsection{The Coefficient of Relative Risk Aversion}

The distinction between absolute and relative risk aversion lies in the size of the hypothetical gamble faced by the household. If the household faces a one-shot gamble of size $A_{t}$ in period $t$,

$$
a_{t+1}=\left(1+r_{t}\right) a_{t}+w_{t} l_{t}+d_{t}-c_{t}+A_{t} \sigma \varepsilon_{t+1}
$$

or the household can pay a one-time fee $A_{t} \mu$ in period $t$ to avoid this gamble, then it follows from Proposition 1 that $\lim _{\sigma \rightarrow 0} 2 \mu(\sigma) / \sigma^{2}$ for this gamble is given by

$$
A_{t} R^{a}\left(a_{t} ; \theta_{t}\right)
$$

The natural definition of $A_{t}$, considered by Arrow (1964) and Pratt (1965), is the household's wealth at time $t$. The gamble in (21) is then over a fraction of the household's wealth and (22) is referred to as the household's coefficient of relative risk aversion.

In models with labor, however, household wealth can be more difficult to define because of the presence of human capital. There are two natural definitions of human capital in these models, leading to two measures of household wealth $A_{t}$ and hence two coefficients of relative risk aversion (22). Note that the household's financial assets $a_{t}$ is not a good measure of wealth $A_{t}$, because $a_{t}$ for an individual household may be zero or negative at some points in time.

When the household's time endowment is not well-defined, such as when $u\left(c_{t}, l_{t}\right)=c_{t}^{1-\gamma} /(1-$ $\gamma)-\eta l_{t}^{1+\chi}$ and no upper bound $\bar{l}$ on $l_{t}$ is specified, or $\bar{l}$ is specified but is arbitrary, it is most natural to define human capital as the present discounted value of labor income, $w_{t} l_{t}^{*}$. Equivalently, total household wealth $A_{t}$ equals the present discounted value of consumption, which follows from the budget constraint (1)-(2). This leads to the following definition:

Definition 2. Let $\left(a_{t} ; \theta_{t}\right)$ be an interior point of $X$. The household's consumption-wealth coefficient of relative risk aversion, denoted $R^{c}\left(a_{t} ; \theta_{t}\right)$, is given by (22) with wealth $A_{t}=A_{t}^{c} \equiv$ $\left(1+r_{t}\right)^{-1} E_{t} \sum_{\tau=t}^{\infty} m_{t, \tau} c_{\tau}^{*}$, the present discounted value of household consumption, where $m_{t, \tau}$ denotes the stochastic discount factor $\prod_{s=t}^{\tau-1} m_{s+1}$, and $m_{s+1}$ is given by (37).

The factor $\left(1+r_{t}\right)^{-1}$ in the definition expresses wealth $A_{t}^{c}$ in beginning- rather than end-of-period- $t$ units, so that in steady state $A^{c}=c / r$ and $R^{c}(a ; \theta)$ is given by

$$
R^{c}(a ; \theta)=\frac{-A^{c} V_{11}(a ; \theta)}{V_{1}(a ; \theta)}+\alpha \frac{A^{c} V_{1}(a ; \theta)}{V(a ; \theta)}=\frac{-u_{11}+\lambda u_{12}}{u_{1}} \frac{c}{1+w \lambda}+\alpha \frac{c u_{1}}{u} .
$$

Alternatively, when the household's time endowment $\bar{l}$ is well specified, one can define human capital to be the present discounted value of the household's time endowment, $w_{t} \bar{l}$. Equivalently, total household wealth $A_{t}$ equals the present discounted value of leisure $w_{t}\left(\bar{l}-l_{t}^{*}\right)$ plus consumption $c_{t}^{*}$, from (1)-(2). This corresponds to the following definition: 
Definition 3. Let $\left(a_{t} ; \theta_{t}\right)$ be an interior point of $X$. The household's consumption-and-leisurewealth coefficient of relative risk aversion, denoted $R^{c l}\left(a_{t} ; \theta_{t}\right)$, is given by $(22)$ with wealth $A_{t}=$ $A_{t}^{c l} \equiv\left(1+r_{t}\right)^{-1} E_{t} \sum_{\tau=t}^{\infty} m_{t, \tau}\left(c_{\tau}^{*}+w_{\tau}\left(\bar{l}-l_{\tau}^{*}\right)\right)$.

In steady state, $A^{c l}=(c+w(\bar{l}-l)) / r$, and

$$
R^{c l}(a ; \theta)=\frac{-u_{11}+\lambda u_{12}}{u_{1}} \frac{c+w(\bar{l}-l)}{1+w \lambda}+\alpha \frac{(c+w(\bar{l}-l)) u_{1}}{u} .
$$

Since leisure is positive, $R^{c}\left(a_{t} ; \theta_{t}\right)<R^{c l}\left(a_{t} ; \theta_{t}\right)$ because the size of the gamble is smaller. The closed-form expressions (23)-(24) are also closely related, differing only by the ratio of the two gambles, $(c+w(\bar{l}-l)) / c .^{18}$

For expositional purposes, define

$$
R^{f l}(a ; \theta) \equiv \frac{-c u_{11}}{u_{1}}+\alpha \frac{c u_{1}}{u}
$$

the coefficient of relative risk aversion in the corresponding model where labor is held exogenously fixed (see Example 1, below). $R^{f l}$ thus ignores or assumes away the household's ability to offset shocks to portfolio values by varying labor supply. By Corollary $4, R^{c}(a ; \theta) \leq R^{f l}(a ; \theta)$. However, $R^{c l}(a ; \theta)$ may be greater or less than $R^{f l}(a ; \theta)$, depending on the importance of leisure in the household's total consumption bundle.

\subsection{Examples}

Example 1. Following Epstein and Zin (1989) and Weil (1989), consider the case where utility depends only on consumption,

$$
u\left(c_{t}, l_{t}\right)=\frac{c_{t}^{1-\gamma}}{1-\gamma}
$$

with $\gamma>0, c_{t} \geq 0$, and $l_{t}$ fixed exogenously at some $l \in \mathbb{R}$ for all $t .^{19}$ Leisure is arbitrary in this example - any $\bar{l}>l$ is observationally equivalent-so $R^{\text {cl }}$ from Definition 3 is not well-defined. Thus, attention is restricted to $R^{c}$ from Definition 2,

$$
R^{c}(a ; \theta)=\frac{-c u_{11}}{u_{1}}+\alpha \frac{c u_{1}}{u}=\gamma+\alpha(1-\gamma)
$$

which motivates the definition of $R^{f l}$ given above. Note that if the household's generalized value function is written using specification (5) rather than (4), with $\rho \equiv 1-\gamma$, then $1-\widetilde{\alpha}=\gamma+\alpha(1-\gamma)$

\footnotetext{
${ }^{18}$ Both Definitions 2 and 3 represent a proper generalization of the traditional definition of risk aversion in an endowment economy. First, both definitions reduce to $R^{f l}$, defined below, when there is no labor in the model. Second, in steady state the household consumes exactly the flow of income from its wealth, $r A$, consistent with standard permanent income theory (where one must include the value of leisure $w(\bar{l}-l)$ as part of consumption when the value of leisure is included in wealth).

${ }^{19}$ In this example, Assumptions 1-8 need to be modified in a straightforward way to the one-dimensional case.
} 
and $R^{c}(a ; \theta)=1-\widetilde{\alpha}$. This is the usual definition of risk aversion for generalized recursive preferences in an endowment economy.

Example 2. Following van Binsbergen et al. (2012), among others ${ }^{20}$ a natural way to incorporate leisure into the preferences in $(26)$ is to let

$$
u\left(c_{t}, l_{t}\right)=\frac{\left(c_{t}^{\chi}\left(1-l_{t}\right)^{1-\chi}\right)^{1-\gamma}}{1-\gamma},
$$

where $\gamma>0, \chi \in(0,1), c_{t}>0$, and $l_{t} \in(0,1) .{ }^{21}$ In this example, the household can be regarded as consuming a single, composite good in each period formed from the Cobb-Douglas aggregate of consumption and leisure. A natural definition of risk aversion is thus $\gamma+\alpha(1-\gamma)=1-\widetilde{\alpha}$, the coefficient of relative risk aversion from Example 1 applied to the single, composite good. Indeed, this is the definition used by van Binsbergen et al. (2012). It is also the value implied by Definition 3 of the present paper, which includes the value of leisure in household wealth:

$$
R^{c l}(a ; \theta)=\frac{-u_{11}+\lambda u_{12}}{u_{1}} \frac{c+w(1-l)}{1+w \lambda}+\alpha \frac{(c+w(1-l)) u_{1}}{u}=\gamma+\alpha(1-\gamma) .
$$

The consumption-wealth coefficient of relative risk aversion from Definition $2, R^{c}$, excludes leisure from household wealth and thus is less than (29), corresponding to the smaller size of the gamble:

$$
R^{c}(a ; \theta)=\frac{-u_{11}+\lambda u_{12}}{u_{1}} \frac{c}{1+w \lambda}+\alpha \frac{c u_{1}}{u}=\gamma \chi+\alpha(1-\gamma) \chi .
$$

In this example, the Cobb-Douglas functional form implies $R^{c}(a ; \theta)=\chi R^{c l}(a ; \theta) .{ }^{22}$ The next section compares these two risk aversion measures to the risk premia on assets.

Note that neither (29) nor (30) corresponds to the fixed-labor measure of risk aversion, $R^{f l}(a ; \theta)=\frac{-c u_{11}}{u_{1}}+\alpha \frac{c u_{1}}{u}=(1-\chi(1-\gamma))+\alpha(1-\gamma)$, a point emphasized by Swanson (2012) for the case of expected utility, $\alpha=0$. The fixed-labor measure $R^{f l}$ ignores the household's ability to offset shocks to portfolio values by varying its hours of work; as a result, $R^{f l}$ does not generally correspond to the household's willingness to hold a risky asset and thus is not closely related to the equilbrium prices of such assets, a fact that will be verified in the next section.

Finally, several other authors consider an alternative parameterization of $(28),{ }^{23}$

$$
u\left(c_{t}, l_{t}\right)=\frac{\left(c_{t}\left(1-l_{t}\right)^{\nu}\right)^{1-\gamma}}{1-\gamma},
$$

\footnotetext{
20 See also Andreasen (2012, 2013), Gourio (2013), Colacito and Croce (2012), and Dew-Becker (2012).

${ }^{21}$ When $\gamma<1$, then $u>0$, risk aversion is increasing in $\alpha$, and $\alpha>0$ corresponds to preferences that are more risk averse than expected utility. When $\gamma>1$, then $u<0$, risk aversion is decreasing in $\alpha$, and $\alpha<0$ corresponds to preferences that are more risk averse than expected utility.

22 That is, $c /(c+w(1-l))=\chi$. One might be surprised that $R^{c}(a ; \theta) \rightarrow 0$ as $\chi \rightarrow 0$. However, as $\chi \rightarrow 0$, $w / c \rightarrow \infty$, so consumption becomes trivial to insure with tiny variations in labor supply.

23 See Gourio (2012), Uhlig (2007), Backus, Routledge, and Zin (2008), and Kung (2012).
} 
where $\gamma>0, \nu>0, c_{t}>0, l_{t} \in(0,1)$, and $\gamma>\nu /(1+\nu)$ for concavity. For this parameterization, $R^{f l}(a ; \theta)=\gamma+\alpha(1-\gamma)=1-\widetilde{\alpha}$, but Definitions $2-3$ of the present paper recognize the household's ability to self-insure itself with variations in hours worked, and imply

and

$$
R^{c}(a ; \theta)=\left(\frac{\nu}{1+\nu}-(1-\gamma) \nu\right)+\alpha(1-\gamma) \nu
$$

$$
R^{c l}(a ; \theta)=(1-(1-\gamma)(1+\nu))+\alpha(1-\gamma)(1+\nu)
$$

Example 3. Following Rudebusch and Swanson (2009), consider the additively separable period utility function

$$
u\left(c_{t}, l_{t}\right)=\frac{c_{t}^{1-\gamma}}{1-\gamma}-\eta \frac{l_{t}^{1+\chi}}{1+\chi},
$$

where $\chi>0, \eta>0, c_{t}>0, l_{t}>0$, and $\gamma>1 .^{24}$ Leisure is essentially arbitrary in this example, since different assumptions regarding $\bar{l}$ have essentially no effect on the equilibrium. Thus, $R^{c l}(a ; \theta)$ is not well-defined, and attention is restricted to $R^{c}(a ; \theta)$ from Defintion 2 ,

$$
R^{c}(a ; \theta)=\frac{\gamma}{1+\frac{\gamma}{\chi} \frac{w l}{c}}+\frac{\alpha(1-\gamma)}{1+\frac{\gamma-1}{1+\chi} \frac{w l}{c}} .
$$

As in Swanson (2012), one can simplify (35) a bit further by assuming $c \approx w l$, an assumption made in this paragraph only and nowhere else in the paper. ${ }^{25}$ In this case,

$$
R^{c}(a ; \theta) \approx \frac{\gamma}{1+\frac{\gamma}{\chi}}+\frac{\alpha(1-\gamma)}{1+\frac{\gamma-1}{1+\chi}} .
$$

Equation (36) is less than $R^{f l}(a ; \theta)=\gamma+\alpha(1-\gamma)$, by an amount that can be dramatic if either of the denominators in (36) is large. Alternatively, as $\chi \rightarrow \infty$, the household's labor margin becomes inflexible and $R^{c} \rightarrow R^{f l}$.

\section{Risk Aversion and Asset Pricing}

As discussed above, the household's aversion to gambles over asset values or wealth depends on its ability to offset the outcome of those gambles with changes in hours worked. In this section, the analysis is extended to show the relationship between risk aversion and risk premia in the LucasBreeden stochastic discounting framework. Risk premia in this framework are closely related to the definition of risk aversion in the present paper, and are generally unrelated to traditional measures of risk aversion that hold household labor fixed.

\footnotetext{
24 The last restriction ensures consistency with Assumption 2. Alternatively, one could assume restrictions on the domain $\Omega$ such that $u(\cdot, \cdot)<0$ for all $\left(c_{t}, l_{t}\right) \in \Omega$. Under either of these assumptions, $u<0$, risk aversion is decreasing in $\alpha$, and $\alpha<0$ corresponds to preferences that are more risk averse than expected utility.

${ }^{25}$ In steady state, $c=r a+w l+d$, so $c=w l$ holds exactly if there is neither capital nor transfers in the model. In any case, $r a+d$ is typically small, since $r \approx .01$.
} 


\subsection{The Stochastic Discount Factor, Risk Premia, and Risk Aversion}

For generalized recursive preferences (4) with labor, Rudebusch and Swanson (2012) show that the household's stochastic discount factor is given by

$$
m_{t+1} \equiv \beta \frac{u_{1}\left(c_{t+1}^{*}, l_{t+1}^{*}\right)}{u_{1}\left(c_{t}^{*}, l_{t}^{*}\right)} \frac{V\left(a_{t+1}^{*} ; \theta_{t+1}\right)^{-\alpha}}{\left(E_{t} V\left(a_{t+1}^{*} ; \theta_{t+1}\right)^{1-\alpha}\right)^{-\alpha /(1-\alpha)}} .
$$

Let $p_{t}^{i}$ denote the ex-dividend time- $t$ price of an asset $i$ that pays stochastic dividend $d_{t}^{i}$ each period. In equilibrium, $p_{t}^{i}$ satisfies

$$
p_{t}^{i}=E_{t} m_{t+1}\left(d_{t+1}^{i}+p_{t+1}^{i}\right)
$$

Let $1+r_{t+1}^{i}$ denote the realized gross return on the asset,

$$
1+r_{t+1}^{i} \equiv \frac{d_{t+1}^{i}+p_{t+1}^{i}}{p_{t}^{i}}
$$

and define the risk premium on the asset, $\psi_{t}^{i}$, to be its expected excess return,

$$
\psi_{t}^{i} \equiv E_{t} r_{t+1}^{i}-r_{t+1}^{f}
$$

where $1+r_{t+1}^{f} \equiv 1 / E_{t} m_{t+1}$ denotes the risk-free rate. Then

$$
\begin{aligned}
\psi_{t}^{i} & =\frac{E_{t} m_{t+1} E_{t}\left(d_{t+1}^{i}+p_{t+1}^{i}\right)-E_{t} m_{t+1}\left(d_{t+1}^{i}+p_{t+1}^{i}\right)}{p_{t}^{i} E_{t} m_{t+1}} \\
& =\frac{-\operatorname{Cov}_{t}\left(m_{t+1}, r_{t+1}^{i}\right)}{E_{t} m_{t+1}}
\end{aligned}
$$

where $\mathrm{Cov}_{t}$ denotes the covariance conditional on information at time $t$.

Intuitively, one can start to see the close relationship between the risk premium and risk aversion as follows. Since $u_{1}\left(c_{t}^{*}, l_{t}^{*}\right)=V_{1}\left(a_{t} ; \theta_{t}\right) /\left(1+r_{t}\right)$,

$$
m_{t+1}=\beta \frac{V_{1}\left(a_{t+1}^{*} ; \theta_{t+1}\right)}{V_{1}\left(a_{t} ; \theta_{t}\right)} \frac{V\left(a_{t+1}^{*} ; \theta_{t+1}\right)^{-\alpha}}{\left(E_{t} V\left(a_{t+1}^{*} ; \theta_{t+1}\right)^{1-\alpha}\right)^{-\alpha /(1-\alpha)}} \frac{1+r_{t}}{1+r_{t+1}}
$$

Then, to first order around the steady state, conditional on information at time $t$,

$$
\begin{aligned}
d m_{t+1} & =\beta \frac{V_{11} d a_{t+1}^{*}+V_{12} d \theta_{t+1}}{V_{1}}-\alpha \beta \frac{V_{1} d a_{t+1}^{*}+V_{2} d \theta_{t+1}}{V}-\beta \frac{d r_{t+1}}{1+r} \\
& =-\beta R^{a}(a ; \theta) d a_{t+1}^{*}+\left(\frac{\beta V_{12}}{V_{1}}-\frac{\alpha \beta V_{2}}{V}\right) d \theta_{t+1}-\beta \frac{d r_{t+1}}{1+r}
\end{aligned}
$$


assuming $V$ is differentiable with respect to $\theta$ at the steady state, and where $d x_{t} \equiv x_{t}-x$, the time- $t$ deviation of variable $x$ from steady state. It follows that

$$
\begin{aligned}
\psi_{t}^{i} \approx R^{a}(a ; \theta) & \operatorname{Cov}_{t}\left(d r_{t+1}^{i}, d a_{t+1}^{*}\right) \\
& +\left(\frac{-V_{12}}{V_{1}}+\alpha \frac{V_{2}}{V}\right) \operatorname{Cov}_{t}\left(d r_{t+1}^{i}, d \theta_{t+1}\right)+\beta \operatorname{Cov}_{t}\left(d r_{t+1}^{i}, d r_{t+1}\right)
\end{aligned}
$$

near the steady state. In (44), $\psi_{t}^{i}$ increases linearly with $R^{a}$, by an amount that depends on the covariance of the asset return with the household's financial wealth.

However, the decomposition in (44) is problematic for several reasons. First, the covariance involving $d a_{t+1}^{*}$ ignores the household's nonfinancial wealth, such as the present value of future transfers and labor income. Instead, the asset's covariance with nonfinancial wealth is relegated to the second term in (44), since $\theta$ determines the household's current and future wages $w$ and transfers $d$. But this covariance is expressed in terms of the "black box" state variable $\theta$ rather than nonfinancial wealth itself, and the coefficient $\left(\frac{-V_{12}}{V_{1}}+\alpha \frac{V_{2}}{V}\right)$ on this covariance is neither clearly related nor unrelated to risk aversion.

Thus, the following decomposition is ultimately more illuminating, at the cost of being somewhat more complicated to derive. First, the stochastic discount factor (37) can be differentiated at steady state, conditional on information at time $t$, to yield

$$
d m_{t+1}=\frac{\beta}{u_{1}}\left[u_{11} d c_{t+1}^{*}+u_{12} d l_{t+1}^{*}\right]-\frac{\alpha \beta}{V} d V_{t+1}
$$

to first order. From the household's intratemporal optimality condition (13),

$$
d l_{t+1}^{*}=-\lambda d c_{t+1}^{*}-\frac{u_{1}}{u_{22}+w u_{12}} d w_{t+1}
$$

to first order. Note that there is an additional term in (46) relative to (14) because $\theta$ (and hence $w, r$, and $d$ ) will generally change in response to macroeconomic shocks.

The corresponding expression for $d c_{t+1}^{*}$ is more complicated and is stated as a lemma:

Lemma 5. To first order in a neighborhood of the nonstochastic steady state,

$$
\begin{aligned}
d c_{t+1}^{*}= & \frac{r}{1+w \lambda}\left[d a_{t+1}+E_{t+1} \sum_{k=1}^{\infty} \frac{1}{(1+r)^{k}}\left(l d w_{t+k}+d d_{t+k}+a d r_{t+k}\right)\right] \\
& +\frac{u_{1} u_{12}}{u_{11} u_{22}-u_{12}^{2}} d w_{t+1}+\frac{-u_{1}}{u_{11}-\lambda u_{12}} E_{t+1} \sum_{k=1}^{\infty} \frac{1}{(1+r)^{k}}\left(\frac{r \lambda}{1+w \lambda} d w_{t+k}-\beta d r_{t+k+1}\right) .
\end{aligned}
$$

Proof: The expression follows from the household's Euler equation, budget constraint, and equation (46). See the Appendix for details. 
Note that if $w, r$, and $d$ are held constant, as in the Arrow-Pratt gamble for a single household in Section 3, then equations (46)-(47) reduce to (14) and (17). More generally, (47) includes the effects of changes in $w, r$, and $d$ on the household's desired consumption. The term in square brackets in (47) describes the change in household wealth - including nonfinancial wealth-and thus the first line of (47) describes the wealth effect on consumption. The last line of (47) describes the substitution effect: changes in consumption due to changes in current and future wages and interest rates. ${ }^{26}$

For notational simplicity, let $d \hat{A}_{t+1} \equiv d a_{t+1}+E_{t+1} \sum_{k=1}^{\infty}(1+r)^{-k}\left(l d w_{t+k}+d d_{t+k}+a d r_{t+k}\right)$, the change in household wealth in (47). Then it is straightforward to show:

Lemma 6. To first order in a neighborhood of the nonstochastic steady state,

$$
d V_{t+1}=u_{1}(1+r) d \hat{A}_{t+1}
$$

Proof: The expression follows from (6), (46), and (47). See the Appendix for details.

Lemma 6 states that the change in household welfare equals the marginal utility of consumption times the change in household wealth. The factor $1+r$ appears in (48) because a change in beginning-of-period- $t$ assets produces $1+r$ units of extra consumption in period $t$.

Equations (45)-(48) then imply the following decomposition:

Proposition 7. To first order in a neighborhood of the nonstochastic steady state,

$$
d m_{t+1}=-R^{a}(a ; \theta) \beta d \hat{A}_{t+1}+\beta d \Phi_{t+1},
$$

where $d \Phi_{t+1} \equiv E_{t+1} \sum_{k=1}^{\infty}(1+r)^{-k}\left(\beta d r_{t+k+1}-\frac{r \lambda}{1+w \lambda} d w_{t+k}\right)$, the intertemporal substitution term from (47). To second order in a neighborhood of the nonstochastic steady state,

$$
\psi_{t}^{i}=R^{a}(a ; \theta) \operatorname{Cov}_{t}\left(d r_{t+1}^{i}, d \hat{A}_{t+1}\right)-\operatorname{Cov}_{t}\left(d r_{t+1}^{i}, d \Phi_{t+1}\right) .
$$

Proof: Substituting (46)-(48) into (45) yields (49). Substituting (49) into (41) yields (50). (Recall that $V=u /(1-\beta)$ and $\beta=E_{t} m_{t+1}$ at steady state.) Finally, $\operatorname{Cov}(d x, d y)$ is accurate to second order when $d x$ and $d y$ are accurate to first order.

The decomposition of the risk premium provided by equation (50) gives a more complete description of the relationship between risk premia and risk aversion than (44). The first term in (50) shows that $\psi_{t}^{i}$ increases locally linearly with $R^{a}$, by an amount that depends on the

\footnotetext{
26 The household's intertemporal elasticity of substitution is given by $-u_{1} /\left(c\left(u_{11}-\lambda u_{12}\right)\right)$, so the last term in (47) describes intertemporal substitution effects on consumption of changes in future wages and interest rates.
} 
covariance between the asset return and the household's wealth, including nonfinancial wealth. This link between risk premia and risk aversion should not be too surprising: Propositions 1-2 described the risk premium for extremely simple, idiosyncratic gambles over household wealth, while Proposition 7 shows that the same coefficient also appears in the household's aversion to more general financial market gambles that may be correlated with aggregate variables such as interest rates, wages, and transfers.

The second term in (50) corresponds to Merton's (1973) "changes in investment opportunities" in the ICAPM framework. Even if $R^{a}=0$ - that is, even if households are risk-neutral in a cross-sectional or CAPM sense- $\psi_{t}^{i}$ can be nonzero. This is because even a risk-neutral household can benefit from an asset that pays off well when the price of the household's total consumption bundle is low. An asset that pays off well when current and future wages are low (and hence leisure is cheap) or current and future interest rates are high (and hence future consumption is cheap) is preferable to an asset that pays off poorly in those situations. Even a risk-neutral household would be willing to pay a premium for such an asset-implying a lower $\psi_{t}^{i}$ —and this effect is captured by the second term in (50).

The fact that households in the present paper face a consumption-leisure tradeoff as well as a current-vs.-future consumption tradeoff implies that the second term in (50) is more general than just changes in the household's investment opportunities. Indeed, the second term in (50) is better described as being due to changes in purchasing opportunities. The decomposition in (50) also suggests that $\psi_{t}^{i}$ is more accurately described as an "expected excess return" rather than a "risk premium" because only the first term in (50) represents compensation to the household for bearing risk; the second term is not compensation for risk but rather reflects the household's ability to take advantage of changes in purchasing opportunities over time.

Finally, the decomposition (50) can be written in terms of relative rather than absolute risk aversion using Definitions $2-3:^{27}$

Corollary 8. In terms of relative risk aversion, the risk premium in (50) can be written as:

$$
\psi_{t}^{i}=R^{c}(a ; \theta) \operatorname{Cov}_{t}\left(d r_{t+1}^{i}, \frac{d \hat{A}_{t+1}}{A^{c}}\right)-\operatorname{Cov}_{t}\left(d r_{t+1}^{i}, d \Phi_{t+1}\right)
$$

or

$$
\psi_{t}^{i}=R^{c l}(a ; \theta) \operatorname{Cov}_{t}\left(d r_{t+1}^{i}, \frac{d \hat{A}_{t+1}}{A^{c l}}\right)-\operatorname{Cov}_{t}\left(d r_{t+1}^{i}, d \Phi_{t+1}\right),
$$

where $A^{c}$ and $A^{\text {cl }}$ are as in Definitions 2-3.

\footnotetext{
${ }^{27}$ Note that $d \hat{A}_{t+1}$ differs slightly from $d A_{t+1}^{c}$ and $d A_{t+1}^{c l}$, which is why (51) and (52) are not written in terms of $d \log A_{t+1}^{c}$ or $d \log A_{t+1}^{c l}$.
} 


\subsection{Numerical Examples}

Two numerical examples help to illustrate the relationship between risk aversion and risk premia derived above. For simplicity, the equity premium is studied in a standard real business cycle (RBC) framework, which provides just enough structure to create an interesting asset pricing problem in which household labor supply can vary endogenously.

The economy consists of a unit continuum of representative households and a unit continuum of perfectly competitive representative firms. Each household has optimization problem (1)-(4) and period utility function to be specified shortly. Each firm has production function

$$
y_{t}=Z_{t} k_{t}^{1-\zeta} l_{t}^{\zeta}
$$

where $y_{t}, k_{t}$, and $l_{t}$ denote firm output, beginning-of-period capital, and labor input, respectively. The productivity parameter $Z_{t}$ follows the exogenous process

$$
\log Z_{t}=\rho_{z} \log Z_{t-1}+\varepsilon_{t}
$$

where $\varepsilon_{t}$ is i.i.d. with mean zero and variance $\sigma_{\varepsilon}^{2}$. Labor and capital are supplied by households at the competitive wage and rental rates $w_{t}$ and $r_{t}^{k}$. Capital is the only asset in the economy that is in nonzero net supply. Households accumulate capital according to

$$
k_{t+1}=\left(1+r_{t}\right) k_{t}+w_{t} l_{t}-c_{t}
$$

where $r_{t} \equiv r_{t}^{k}-\delta, \delta$ is the capital depreciation rate, and $c_{t}$ denotes household consumption.

An equity security is defined to be a claim on the aggregate consumption stream, where aggregate consumption $C_{t}=c_{t}$ in equilibrium. The ex-dividend price of the equity claim, $p_{t}$, satisfies

$$
p_{t}=E_{t} m_{t+1}\left(C_{t+1}+p_{t+1}\right)
$$

in equilibrium, where $m_{t+1}$ is given by (37). The equity premium, $\psi_{t}$, is defined to be the expected excess return

$$
\psi_{t} \equiv \frac{E_{t}\left(C_{t+1}+p_{t+1}\right)}{p_{t}}-\left(1+r_{t}^{f}\right)
$$

Following standard calibrations in the literature, a period in the model is taken to be one quarter in the data, $\beta$ is set to $.99, \delta$ to $.025, \zeta$ to .7 , and $\sigma_{\varepsilon}$ to .01 . The cases $\rho_{z}<1$ and $\rho_{z}=1$ are both considered in the examples below. Once the period utility function is specified, the model is solved using perturbation methods, as in Rudebusch and Swanson (2012) and Swanson (2012). This involves computing a nonstochastic steady state for the model (or transformed 
version of the model) and an $n$ th-order Taylor series approximation to the true nonlinear solution for the model's endogenous variables around the steady state. (Results in the figures below are for $n=5$.) Additional details of the solution algorithm and computer code are provided in the Appendix and in Swanson, Anderson, and Levin (2006). Aruoba, Fernández-Villaverde, and Rubio-Ramírez (2006) solve a standard RBC model using a variety of numerical methods and find that the fifth-order perturbation solution is among the most accurate methods globally as well as being the fastest to compute.

Example 4. Consider the additively separable period utility function from Rudebusch and Swanson (2009) and Example 3,

$$
u\left(c_{t}, l_{t}\right)=\frac{c_{t}^{1-\gamma}}{1-\gamma}-\eta \frac{l_{t}^{1+\chi}}{1+\chi}
$$

Set $\rho_{z}=0.9, \gamma=5, \chi=1.5$, and $\alpha=-10$ as baseline values, and consider how the equity premium and risk aversion vary as each of $\gamma, \chi$, and $\alpha$ are varied in turn. ${ }^{28}$ For each set of parameter values, the model is solved as described above.

Figure 1 plots the equity premium and risk aversion as functions of $\chi, \gamma$, and $\alpha$. The solid black line in each panel graphs the equity premium, $\psi$, against the right axis. The equity premium in this model is very small, less than 25 basis points per year in each of the panels; this is a manifestation of Roewenhorst's (1995) and Lettau and Uhlig's (2000) finding that the equity premium is an even larger puzzle in RBC models with endogenous labor than in an endowment economy, because households can endogenously smooth consumption in response to shocks. The dashed blue line in each panel plots the coefficient of relative risk aversion, $R^{c}(a ; \theta)$ from equation (35), against the left axis. For comparison, the dotted red line in each panel plots the fixed-labor measure of risk aversion for these preferences, $R^{f l}(a ; \theta)=\gamma+\alpha(1-\gamma)$, also against the left axis.

In each of the three panels in Figure 1, the equity premium tracks $R^{c}$ closely, and is essentially unrelated to $R^{f l}$. In the top panel, $R^{f l}$ is independent of $\chi$ and thus is constant at 45 , yet the equity premium varies by a factor of four, along with $R^{c}$. In the middle panel, $R^{f l}$ increases linearly with $\gamma$, ranging from about 1 up to 1090 (values above 32 are off the chart and not depicted), while the equity premium is a concave function of $\gamma$ that corresponds closely to $R^{c}$. In the bottom panel, the equity premium varies about linearly with $\alpha$ and $R^{c}$, but does not

\footnotetext{
${ }^{28}$ To allow for balanced growth or $\rho_{z}=1$, the preference specification (58) would have to be modified, as in Rudebusch and Swanson (2012). For simplicity, those modifications are not considered in this example.
} 

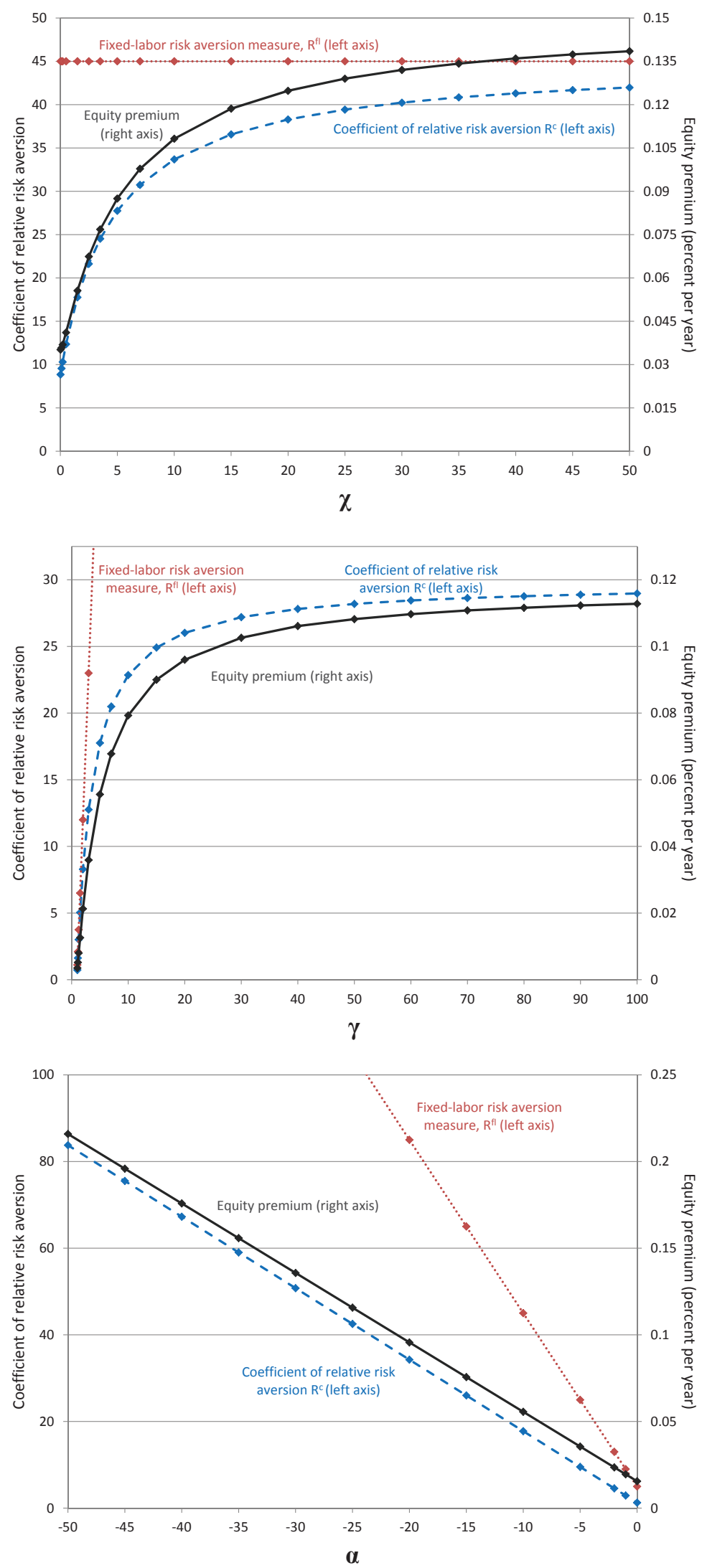

Figure 1. The equity premium and risk aversion in a real business cycle model with generalized recursive preferences and period utility $u\left(c_{t}, l_{t}\right)=c_{t}^{1-\gamma} /(1-\gamma)-\eta l_{t}^{1+\chi} /(1+\chi)$. Solid black lines depict the equity premium, dashed blue lines the coefficient of relative risk aversion $R^{c}$, and dotted red lines the traditional, fixed-labor measure of risk aversion, $R^{f l}=\gamma+\alpha(1-\gamma)=1-\widetilde{\alpha}$. In the top panel, $\chi$ ranges from .01 to 50 while $\gamma$ is fixed at 5 and $\alpha$ at -10 ; in the middle panel, $\gamma$ ranges from 1.01 to 100 while $\chi$ is fixed at 1.5 and $\alpha$ at -10 ; in the bottom panel, $\alpha$ ranges from -50 to 0 while $\chi$ is fixed at 1.5 and $\gamma$ at 5 . In each panel, the equity premium is closely related to $R^{c}$ and is essentially unrelated to $R^{f l}$. See text for details. 
(a) Consumption

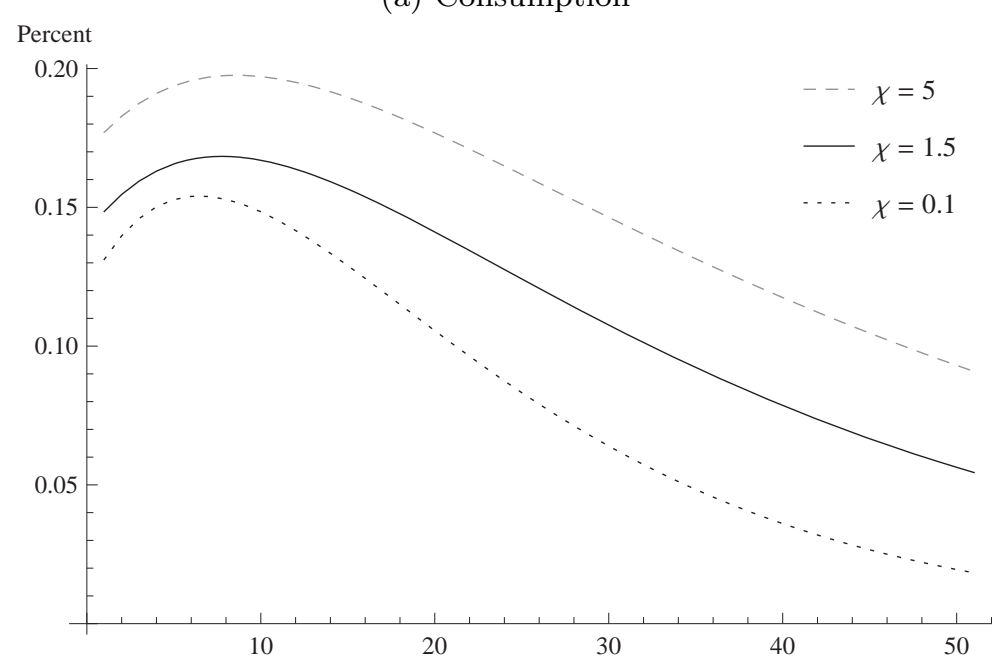

(b) Labor

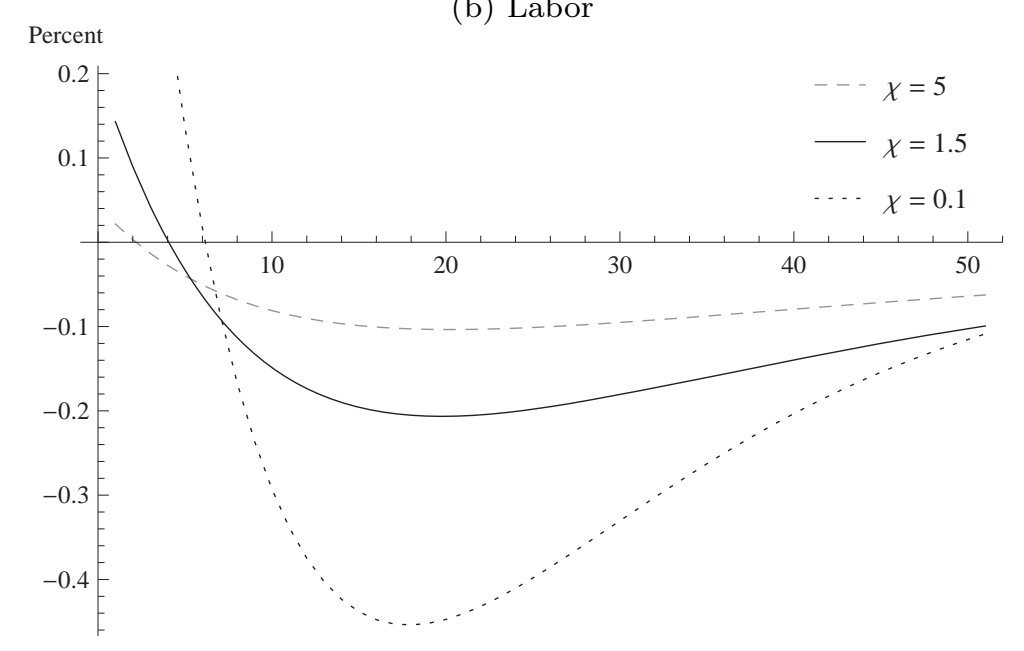

(c) Capital Stock

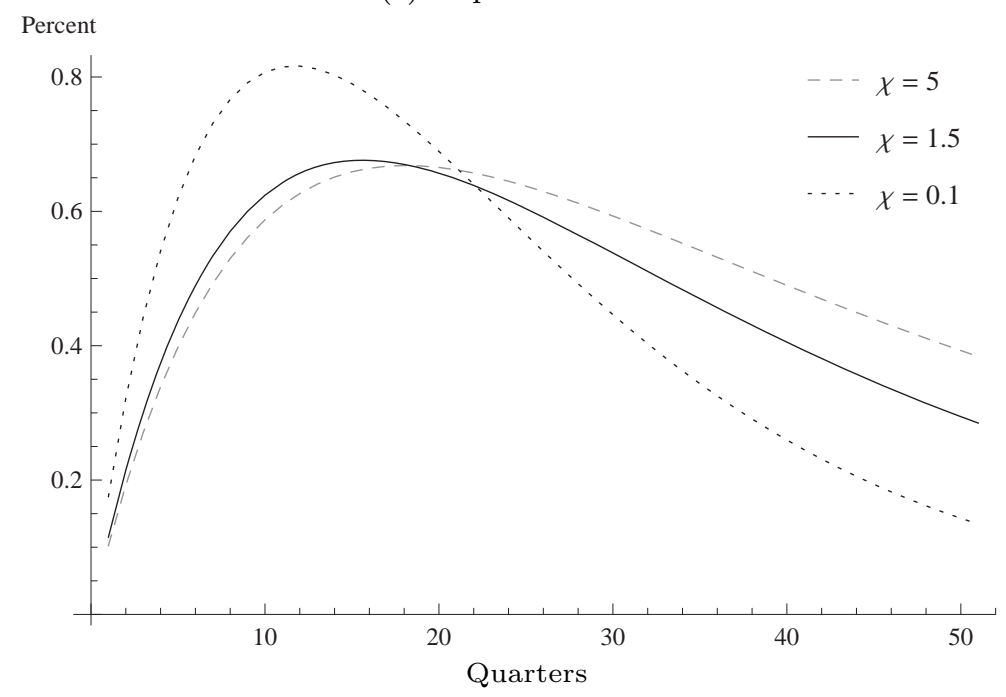

Figure 2. Impulse response functions for (a) consumption, (b) labor, and (c) the capital stock to a $1 \%$ technology shock in the real business cycle model from Example 4 and Figure 1, with generalized recursive preferences and period utility $u\left(c_{t}, l_{t}\right)=c_{t}^{1-\gamma} /(1-\gamma)-\eta l_{t}^{1+\chi} /(1+\chi)$. In each panel, $\gamma=5, \alpha=-10$, and $\chi \in\{0.1,1.5,5\}$. When $\chi$ is lower, the household varies labor supply by more to smooth consumption, even though labor and consumption comove positively in the short run. See text for details. 
correspond to $R^{f l} \cdot{ }^{29}$ Note that, in the bottom panel, more negative values of $\alpha$ imply greater risk aversion because $u \leq 0$; also, the equity premium does not converge to zero as $R^{c} \rightarrow 0$ due to the additional ICAPM term in (50) reflecting changes in purchasing opportunities discussed earlier.

Intuitively, lower values of $\chi$ imply a more flexible labor margin, which gives the household more ability to insure itself from consumption fluctuations. This can be seen clearly in Figure 2, which plots first-order impulse response functions for consumption, labor, and the capital stock to a one percent positive shock to productivity $Z_{t}$. In each panel, the solid black line depicts the impulse response for the baseline parameterization of the model and the dashed and dotted lines plot impulse response functions for the cases $\chi=5$ and $\chi=0.1$, respectively. For all three parameterizations, consumption rises in response to the productivity shock, labor rises on impact and then falls, and household savings increases (as evidenced by the rise in the capital stock). When $\chi$ is lower, the household's labor margin is more flexible and the household reduces labor supply by more, on net, in response to the shock, thereby smoothing consumption. Note how this intuition holds despite the fact that labor initially rises on impact, as a result of the substitution effect on labor supply. Thus, the fact that the short-run correlation between labor and consumption is positive in the model does not prevent the household from using labor supply to smooth its consumption in response to shocks.

Example 5. Consider the Cobb-Douglas preference specification from van Binsbergen et al. (2012) and Example 2,

$$
u\left(c_{t}, l_{t}\right)=\frac{\left(c_{t}^{\chi}\left(1-l_{t}\right)^{1-\chi}\right)^{1-\gamma}}{1-\gamma} .
$$

Following Gourio (2013), set $\rho_{z}=1, \gamma=0.5, \chi=0.3$, and $\alpha=19$, and consider how the equity premium and risk aversion vary as $\chi, \gamma$, and $\alpha$ are varied in turn. ${ }^{30}$ For each set of parameter values, the model is solved as described above.

Figure 3 plots the equity premium and risk aversion as functions of $\chi, \gamma$, and $\alpha$. As in Figure 1, the solid black line in each panel depicts the equity premium, $\psi$, the dashed blue line plots the consumption-wealth coefficient of relative risk aversion, $R^{c}(a ; \theta)$, and the dotted red

\footnotetext{
${ }^{29}$ The equity premium $\psi, R^{c}$, and $R^{f l}$ all vary about linearly with $\alpha$, but the magnitude of $R^{f l}$ does not agree with $\psi$. For example, in the top panel of Figure 1, an equity premium of about $14 \mathrm{bp}$ corresponds to risk aversion around 45 by either measure $R^{c}$ or $R^{f l}$. In the bottom panel of Figure $1, \psi$ of about 15 bp also corresponds to $R^{c}$ of about 45 (at $\alpha \approx-27$ ), but would require $R^{f l} \approx 100$.

Results for the bond premium - the risk premium on a long-term real bond - are essentially the same as those for the equity premium, although the model-implied bond premium is generally smaller than the equity premium and can even be negative if interest rates tend to move countercylically, as discussed in Rudebusch and Swanson (2012). In this and the following example, the magnitude of the bond premium tracks $R^{c}$ closely and does not correspond to $R^{f l}$ or $R^{c l}$.

${ }^{30}$ Gourio sets $1-\widetilde{\alpha}=\gamma+\alpha(1-\gamma)=10$.
} 

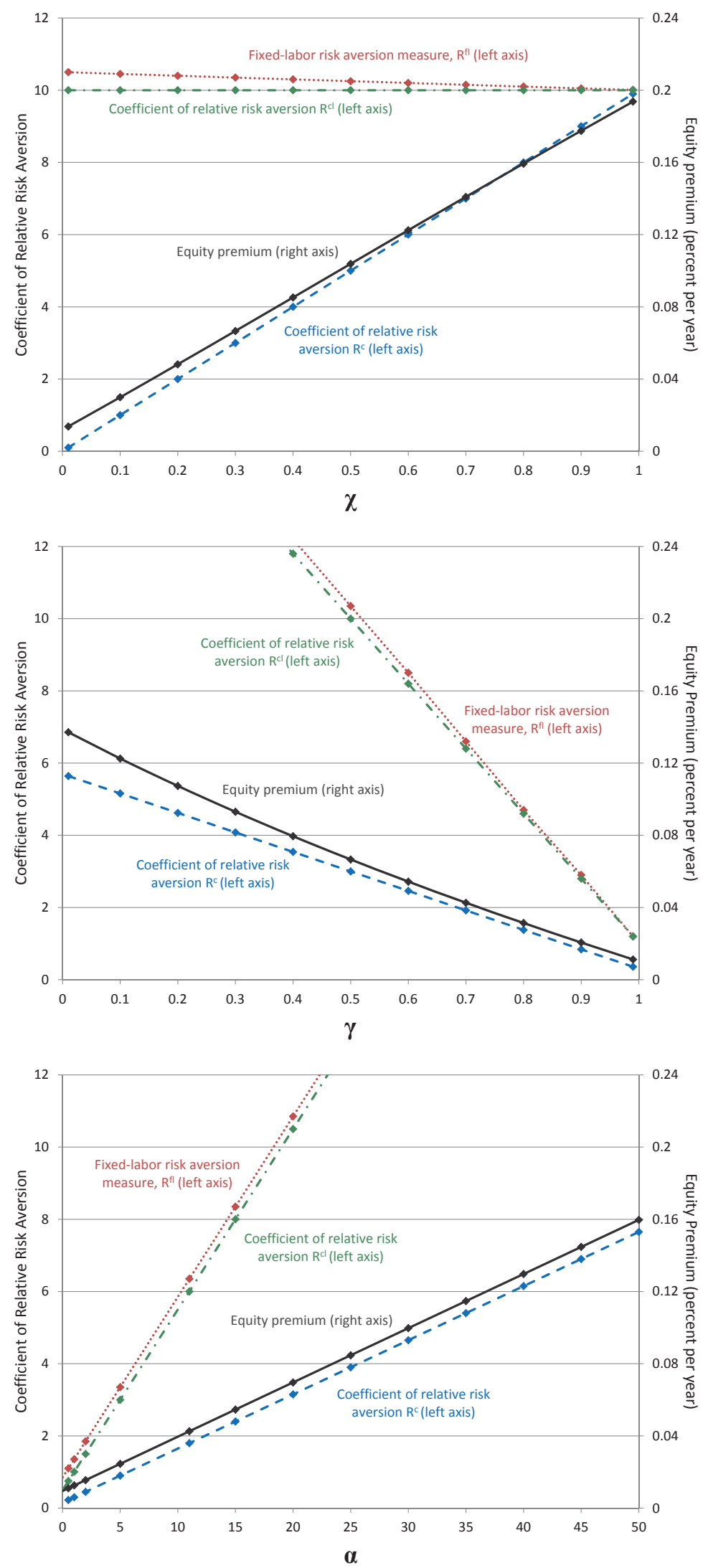

Figure 3. The equity premium and risk aversion in an RBC model with generalized recursive preferences and period utility $u\left(c_{t}, l_{t}\right)=\left(c_{t}^{\chi}\left(1-l_{t}\right)^{1-\chi}\right)^{1-\gamma} /(1-\gamma)$. Solid black lines depict the equity premium, dashed blue lines the coefficient of relative risk aversion $R^{c}$, dotted red lines the fixed-labor measure of risk aversion $R^{f l}$, and dash-dot green lines the coefficient of relative risk aversion $R^{c l}$. In the top panel, $\chi$ ranges from .01 to .99 while $\gamma$ is fixed at 0.5 and $\alpha$ at 19 ; in the middle panel, $\gamma$ ranges from .01 to .99 while $\chi$ is fixed at 0.3 and $\alpha$ at 19; in the bottom panel, $\alpha$ ranges from 0 to 50 while $\chi$ is fixed at 0.3 and $\gamma$ at 0.5 . In each panel, the equity premium is closely related to $R^{c}$ and is essentially unrelated to $R^{f l}$ and $R^{c l}$. See text for details. 
line graphs the traditional, fixed-labor measure, $R^{f l}(a ; \theta)$. As in Figure 1 , the equity premium in Figure 3 tracks $R^{c}$ closely, and is essentially unrelated to $R^{f l}$. In the top panel, $R^{f l}$ is nearly constant at a value of about 10, yet the equity premium varies by a factor of almost ten, along with $R^{c}$. (The equity premium does not converge to zero along with $R^{c}$ due to the additional ICAPM term in (50) reflecting changes in purchasing opportunities.) In the middle panel, $R^{f l}$ increases linearly as $\gamma$ falls, ranging from about 1 up to 19.5 (values above 12 are not depicted), but the equity premium increases at a more moderate pace corresponding to $R^{c}$. For example, a value of $\psi=10 \mathrm{bp}$ is associated with $R^{c} \approx 5$ in the top panel of Figure 3, while a value of $\psi=10 \mathrm{bp}$ in the middle panel requires $R^{c} \approx 5$ vs. $R^{f l} \approx 16$, at $\gamma \approx .2$. In the bottom panel, the equity premium increases about linearly with $\alpha$ and $R^{c}$, while $R^{f l}$ again grows too quickly.

Household leisure is well-defined in this example, so the consumption-and-leisure-wealth coefficient of relative risk aversion, $R^{c l}(a ; \theta)=\gamma+\alpha(1-\gamma)=1-\widetilde{\alpha}$, is depicted in Figure 3 as the dash-dotted green line. Perhaps surprisingly, $R^{c l}$ is not closely related to the equity premium $\psi$. In the top panel of Figure $3, R^{c l}$ is independent of $\chi$ and thus constant at 10 , while $\psi$ varies by a factor of almost ten. In the middle and bottom panels, $R^{c l}$ grows linearly along with $R^{f l}$ at a rate much greater than $\psi$. The reasons for the divergence between $R^{c l}$ and the equity premium are discussed in more detail below.

\subsection{Relative Risk Aversion $R^{c}$ vs. $R^{c l}$ and the Equity Premium}

It may seem surprising that $R^{c l}$ is not more closely related to the equity premium in Figure 3 , given the composite good interpretation for consumption and leisure for those preferences. Instead, the consumption-wealth risk aversion coefficient, $R^{c}$, provides the better measure. Looking at the decomposition of the equity premium provided by Corollary 8, what Figure 3 is saying is that the covariance $\operatorname{Cov}_{t}\left(d r_{t+1}^{i}, d \hat{A}_{t+1} / A^{c}\right)$ is much closer to being invariant with respect to changes in the household's preference parameters than is the covariance $\operatorname{Cov}_{t}\left(d r_{t+1}^{i}, d \hat{A}_{t+1} / A^{c l}\right) .{ }^{31}$ In this section, the reasons for this result are explored and discussed.

Note first that — unlike the traditional, fixed-labor measure $R^{f l}$ — both $R^{c}$ and $R^{c l}$ recognize that households will vary their labor supply to insure themselves from portfolio fluctuations. The issue here is simply whether the value of leisure should be included in household wealth when measuring relative risk aversion, with $R^{c l}$ including the value of leisure and $R^{c}$ excluding it.

\footnotetext{
${ }^{31}$ As will be discussed below, the second covariance term $\operatorname{Cov}_{t}\left(d r_{t+1}^{i}, d \Phi_{t+1}\right)$ in Corollary 8 does not vary much with changes in the household's preference parameters.
} 
In a model with two consumption goods (and no labor) and period utility $u\left(c_{1 t}, c_{2 t}\right)=$ $\left(c_{1 t}^{\chi} c_{2 t}^{1-\chi}\right)^{1-\gamma} /(1-\gamma)$, it would seem bizarre to equate household wealth to the present value of consumption of one of the goods, excluding the value of the other. Yet that is essentially what the results in Figure 3 and Example 5 suggest.

The key difference in Example 5 is that consumption and leisure appear separately elsewhere in the model (e.g., in the production function), which is inconsistent with the composite good interpretation. In a model with two consumption goods, varying the parameter $\chi$ between 0 and 1 might change the relative sizes of the two consumption good sectors in steady state, but would not have any aggregate general equilibrium implications. In contrast, varying the parameter $\chi$ in Example 5 has important general equilibrium effects on steady-state capital, labor, wealth, and other aggregate variables. ${ }^{32}$

To see the effects of $\chi$ on the steady state and the covariance term $\operatorname{Cov}_{t}\left(d r_{t+1}^{i}, d \hat{A}_{t+1}\right)$ in Example 5, start by computing the model's steady state. The steady-state interest rate $r=$ $(1-\beta) / \beta$ and marginal product of capital $r^{k}=(1-\zeta) y / k$, so the output-capital ratio satisfies

$$
\frac{y}{k}=\frac{1}{1-\zeta}\left(\frac{1-\beta}{\beta}+\delta\right)
$$

From the production function, $(l / k)=(y / k)^{1 / \zeta}$, and the aggregate resource constraint implies $(c / k)=(y / k)+\delta$. Thus, the ratios $y / k, l / k$, and $c / k$ are all invariant with respect to $\chi$, and so is the steady-state wage $w=\zeta \frac{(y / k)}{(l / k)}$. Finally, the household's period utility function implies $\chi w(1-l)=(1-\chi) c$, and thus

$$
k=\frac{w}{w(l / k)+\frac{1-\chi}{\chi}(c / k)} .
$$

The wage $w$ and ratios $l / k$ and $c / k$ are invariant with respect to $\chi$, so the aggregate equilibrium level of $k$ is increasing in $\chi$, ranging from 0 to $(y / k)^{-1 / \zeta}$ as $\chi$ ranges from 0 to 1.

Thus, varying the parameter $\chi$ in Example 5 changes not just the composition of the consumption-leisure aggregate good, but also the equilibrium levels of $k$ and household wealth $A^{c}$ and $A^{c l}$, among other variables. This, in turn, changes the crucially important covariance $\operatorname{Cov}_{t}\left(d r_{t+1}^{i}, d \hat{A}_{t+1}\right)$ in Proposition 7. In particular, $\operatorname{Cov}_{t}\left(d r_{t+1}^{i}, d \hat{A}_{t+1}\right)$ is roughly proportional

\footnotetext{
32 In partial equilibrium, the interpretation of consumption and leisure as a composite good for the household in Example 5 is valid. The issue is that the composite good interpretation is not valid in the general equilibrium of the model and the graphs in Figure 3 plot the general equilibrium relationship between the equity premium (or risk aversion) and the parameters $\chi, \gamma$, and $\alpha$.
} 
to steady-state $k$, because $d \hat{A}_{t+1}=d a_{t+1}+E_{t+1} \sum_{k=1}^{\infty}(1+r)^{-k}\left(l d w_{t+k}+a d r_{t+k}\right)$ scales about linearly with $k .^{33}$

Finally, household wealth $A^{c}$ is proportional to $k \cdot{ }^{34}$ As a result, $\operatorname{Cov}_{t}\left(d r_{t+1}^{i}, d \hat{A}_{t+1} / A^{c}\right)$ in Corollary 8 is roughly invariant with respect to $\chi$, implying a tight, linear relationship between $R^{c}(a ; \theta)$ and the equity premium $\psi \cdot{ }^{35}$ This close relationship is clearly visible in Figure 3.

By contrast, $A^{c l}$, the leisure-inclusive measure of household wealth, is not proportional to $k$. The value of leisure, $w(1-l)$, decreases with $k$ (because $w$ is invariant and $1-l$ decreases), while nonhuman wealth increases with $k$. As a result, $A^{c l}$ has no simple relationship to $k$ and $\operatorname{Cov}_{t}\left(d r_{t+1}^{i}, d \hat{A}_{t+1} / A^{c l}\right)$ varies substantially with changes in $\chi$. Thus, there is no stable relationship between $R^{c l}$ and the equity premium in Corollary 8 and Example 5 , as is evident in Figure 3.

Intuitively, consumption and leisure do not form a true composite good in the model because labor appears separately in the production function. Thus, a composite-good measure of risk aversion $R^{c l}$ is not necessarily the best measure and in fact does not match the equity premium in Figure 3. Instead, the consumption-wealth coefficient of relative risk aversion, $R^{c}$ — which recognizes the household's flexible labor margin but excludes the value of leisure from total household wealth - seems to be more closely related to the equity premium.

Of course, the equity premium depends not just on $R^{c}$ but also on the two covariance terms in Corollary 8 - the covariance of the equity return with household wealth and with changes in purchasing opportunities. To the extent that these covariances change as parameters of any given model are varied, the relationship between the equity premium and $R^{c}$ will be weaker. However, for standard macroeconomic models like those considered in this section, the risk aversion measure $R^{c}$ seems to provide a good benchmark.

\section{Risk Aversion Away from the Steady State}

The closed-form expressions for risk aversion derived in Section 3 hold exactly only at the model's nonstochastic steady state. For values of $\left(a_{t} ; \theta_{t}\right)$ away from steady state, these expressions are only

\footnotetext{
${ }^{33}$ Household assets $a=k$ and the ratio $l / k$ is constant, so $a$ and $l$ scale linearly with $k$. (Labor scales linearly up to its maximum value $l=1$, which is attained when $\chi=1$ and $k=1 /(l / k)$.) In contrast, $d r_{t+1}$ and $d w_{t+1}$ hardly change with $k$ because the marginal products of capital and labor, $(1-\zeta) y_{t} / k_{t}$ and $\zeta y_{t} / l_{t}$, are invariant to changes in steady-state $k$. The term $d a_{t+1}$ grows about linearly with $k$ because technology shocks in the model are multiplicative, so the effects of technology shocks scale. Thus, $d \hat{A}_{t+1}$ scales about linearly with $k$. The return $r_{t+1}^{i}$ on the consumption claim hardly changes with $k$ because both sides of the household's Euler equation scale linearly with $k$. Thus, $\operatorname{Cov}_{t}\left(d r_{t+1}^{i}, d \hat{A}_{t+1}\right)$ varies roughly linearly with $k$.

${ }^{34}$ Because consumption and hence the present discounted value of consumption scale linearly with $k$.

35 The second covariance term in Corollary $8, \operatorname{Cov} t\left(d r_{t+1}^{i}, d \Phi_{t+1}\right)$, is not strictly invariant to changes in $\chi$, but this term is much smaller than the first and thus does not have a substantial effect on $\psi$ in Figure 3.
} 

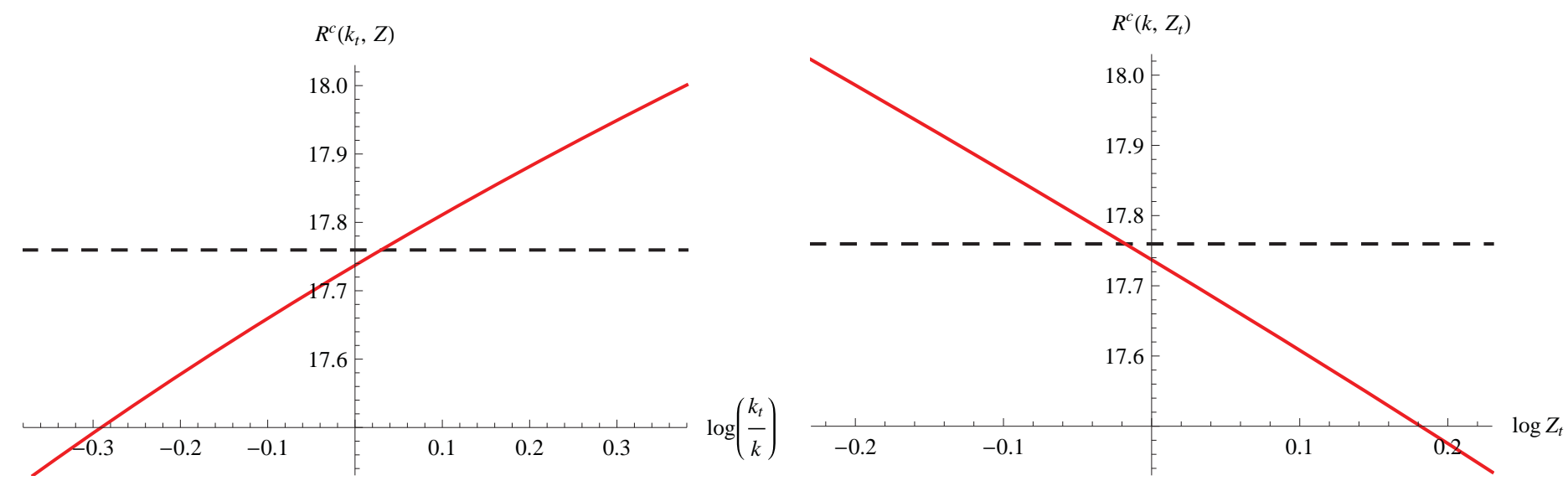

Figure 4. Coefficient of relative risk aversion $R^{c}$ as a function of the state $\left(k_{t} ; Z_{t}\right)$ in a real business cycle model with generalized recursive preferences and period utility $u\left(c_{t}, l_{t}\right)=c_{t}^{1-\gamma} /(1-\gamma)-\eta l_{t}^{1+\chi} /(1+\chi)$. Dashed black lines depict the closed-form, steady-state value $R^{c}(k ; Z)$, solid red lines the numerical solution for $R^{c}\left(k_{t} ; Z_{t}\right)$. In the left panel, $\log \left(k_{t} / k\right)$ ranges from -0.38 to 0.38 while $\log Z_{t}$ is fixed at 0 ; in the right panel, $Z_{t}$ ranges from -0.23 to 0.23 while $k_{t}$ is fixed at $k$. In both panels, $R^{c}\left(k_{t} ; Z_{t}\right)$ is close to $R^{c}(k ; Z)$ and never near the traditional, fixed-labor value of $R^{f l}=45$. See text for details.

approximations. In this section, the accuracy of those approximations is evaluated by computing risk aversion numerically away from the steady state for the standard real business cycle model described above.

The setup and parameterization of the model are as described previously. Household preferences are assumed to have the same additively separable form as in Examples 3-4, with parameter values $\gamma=5, \chi=1.5$, and $\alpha=-10$. The state variables of the model are $k_{t}$ and $Z_{t} .{ }^{36}$ The household's consumption-wealth coefficient of relative risk aversion at the steady state, $R^{c}(k ; Z)$, is given by equation (35). For the parameter values above, this implies a risk aversion coefficient of 17.76, a little more than one-third the traditional measure of $1-\widetilde{\alpha}=\gamma+\alpha(1-\gamma)=45$.

For values of $\left(k_{t} ; Z_{t}\right)$ away from the steady state, equations (9) and (11)-(15) remain valid, and can be used to compute $R^{c}\left(k_{t} ; Z_{t}\right)$ numerically. Equations for $R^{c}, V_{1}, V_{11}, \lambda_{t}$, and $\partial c_{t}^{*} / \partial a_{t}$ are appended to the standard set of RBC equilibrium conditions and solved using the same fifthorder perturbation method as in the previous section. (A complete list of equations and additional details regarding the numerical solution algorithm are provided in the Appendix.)

Figure 4 graphs the result as a function of $\log \left(k_{t} / k\right)$ and $\log Z_{t}$ over a wide range of values for these variables, about \pm 10 standard deviations (equal to about \pm 38 percent and \pm 23 percent

\footnotetext{
36 The household's endogenous state variable is its own holdings of capital, $k_{t}$. The exogenous state variables of the model are $Z_{t}$ and the aggregate capital stock, $K_{t}$. Thus, the state vector of the household's optimization problem could be written more precisely as $\left(k_{t} ; Z_{t}, K_{t}\right)$, or even $\left(k_{t} ; Z_{t}, K_{t}, \sigma_{\varepsilon}^{2}\right)$, since the nonstochastic steady state requires setting $\sigma_{\varepsilon}^{2}=0$. However, in equilibrium, $k_{t}=K_{t}$, so for simplicity the state vector in this example is written as $\left(k_{t} ; Z_{t}\right)$.
} 

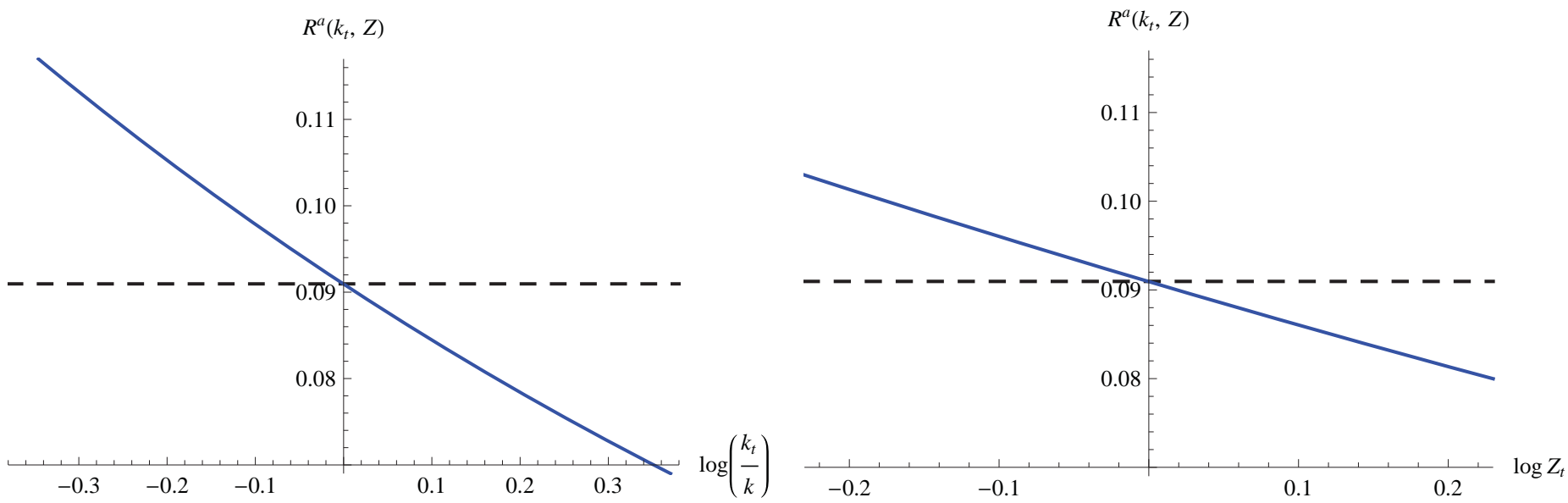

Figure 5. Coefficient of absolute risk aversion $R^{a}$ as a function of the state $\left(k_{t} ; Z_{t}\right)$ in a real business cycle model with generalized recursive preferences and period utility $u\left(c_{t}, l_{t}\right)=c_{t}^{1-\gamma} /(1-\gamma)-\eta l_{t}^{1+\chi} /(1+\chi)$. Dashed black lines depict the closed-form, steady-state value $R^{a}(k ; Z)$, solid blue lines the numerical solution for $R^{a}\left(k_{t} ; Z_{t}\right)$. Absolute risk aversion is decreasing with both $k_{t}$ and $Z_{t}$. See notes to Figure 4 and text for details.

in logarithmic terms for $\log k_{t}$ and $\log Z_{t}$, respectively). ${ }^{37}$ The horizontal dashed black lines in Figure 4 report the constant, closed-form value for risk aversion at the nonstochastic steady state, $R^{c}(k ; Z)$, equal to 17.76. The solid red lines in the figure plot the numerical solution for $R^{c}\left(k_{t} ; Z_{t}\right)$ for general values of $k_{t}$ and $Z_{t} \cdot{ }^{38}$ The key point of Figure 4 is that, even over the very wide range of values of the state variables considered, the household's coefficient of relative risk aversion ranges between about 17.45 and 18 , very close to $R^{c}(k ; Z)$, and never near the traditional, fixedlabor value of $R^{f l}=45$. Thus, the closed-form expressions in Section 3 seem to provide a good approximation to household risk aversion in a standard model even far away from steady state.

It is also interesting that the household's risk aversion is countercyclical with respect to the state variables $k_{t}$ and $Z_{t}$. This can be seen most clearly in Figure 5, which graphs the household's coefficient of absolute risk aversion, $R^{a}\left(k_{t} ; Z_{t}\right)$ over the same range of values for $k_{t}$ and $Z_{t}$ as in Figure 4. The absolute risk aversion coefficient of .09 implies that the household is willing to pay about 9 cents to avoid a fair gamble with a standard deviation of one dollar. This willingness to pay varies from about 7 to 12 cents over the range of values for the state variables in Figure 5, with higher values of the states corresponding to higher household wealth and lower risk aversion.

Looking back at Figure 4, relative risk aversion is not countercyclical in that figure with respect to $k_{t}$ because household wealth — and thus the size of the hypothetical gamble faced by the

\footnotetext{
${ }^{37}$ The unconditional standard deviations of $\log Z_{t}$ and $\log \left(k_{t} / k\right)$ are about 2.3 and 3.8 percent, respectively. The ergodic mean of $\log Z_{t}$ is zero and that of $\log \left(k_{t} / k\right)$ is about .006 , or 0.6 percent.

${ }^{38}$ The red lines do not intersect the black lines at the vertical axis because $c_{t}^{*}$ and $l_{t}^{*}$ evaluated at $k_{t}=k$ and $Z_{t}=Z$ do not equal the nonstochastic steady state values $c$ and $l$ due to the presence of uncertainty (e.g., precautionary savings).
} 
household - is increasing in $k_{t}$ and $Z_{t}$. Indeed, for higher $k_{t}$, the increase in wealth is sufficiently large that the household's relative risk aversion increases with $k_{t}$, even though absolute risk aversion is decreasing.

\section{Balanced Growth}

The analysis in the previous sections has abstracted from growth for simplicity, but the results carry through essentially unchanged to the case of balanced growth. The corresponding expressions are briefly collected in this section and proved in the Appendix.

A detailed discussion of balanced growth is provided in King, Plosser, and Rebelo (1988, 2002). Along a balanced growth path, $x \in\{l, r\}$ satisfies $x_{t+k}=x_{t}$ for $k=1,2, \ldots$, and the time subscript is dropped to denote the constant steady-state value. For $x \in\{a, c, w, d\}, x_{t+k}=G^{k} x_{t}$ for $k=1,2, \ldots$, for some $G \in(0,1+r)$, and $x_{t}^{b g}$ is used to denote the balanced growth path value. The balanced growth path value of $\theta_{t}$ is denoted by $\theta_{t}^{b g}$, although the elements of $\theta$ may grow at different constant rates over time (or remain constant).

Lemma 9. Given Assumptions $1-7$ and $8^{\prime}$, for all $k=1,2, \ldots$ along the balanced growth path: i) $\lambda_{t+k}^{b g}=G^{-k} \lambda_{t}^{b g}$, where $\lambda_{t}^{b g}$ denotes the balanced growth path value of $\lambda_{t}$, ii) $\partial c_{t+k}^{*} / \partial a_{t}=$ $G^{k} \partial c_{t}^{*} / \partial a_{t}$, iii) $\partial l_{t+k}^{*} / \partial a_{t}=\partial l_{t}^{*} / \partial a_{t}$, and $\left.i v\right) \partial c_{t}^{*} / \partial a_{t}=(1+r-G) /\left(1+w_{t}^{b g} \lambda_{t}^{b g}\right)$.

Proof: See Appendix.

Note that $w_{t}^{b g} \lambda_{t}^{b g}$ in Lemma 9 is constant over time because $w$ and $\lambda$ grow at reciprocal rates. The larger is $G$, the smaller is $\partial c_{t}^{*} / \partial a_{t}$, since the household chooses to absorb a greater fraction of asset shocks in future periods.

Proposition 10. Given Assumptions 1-7 and 8', absolute risk aversion, evaluated along the balanced growth path, satisfies

$$
R^{a}\left(a_{t}^{b g} ; \theta_{t}^{b g}\right)=\frac{-V_{11}\left(a_{t+1}^{b g} ; \theta_{t+1}^{b g}\right)}{V_{1}\left(a_{t+1}^{b g} ; \theta_{t+1}^{b g}\right)}+\alpha \frac{V_{1}\left(a_{t+1}^{b g} ; \theta_{t+1}^{b g}\right)}{V\left(a_{t+1}^{b g} ; \theta_{t+1}^{b g}\right)}
$$

and

$$
R^{a}\left(a_{t}^{b g} ; \theta_{t}^{b g}\right)=\frac{-u_{11}+\lambda_{t}^{b g} u_{12}}{u_{1}} \frac{\frac{1+r}{G}-1}{1+w_{t}^{b g} \lambda_{t}^{b g}}+\alpha\left(\frac{1+r}{G}-1\right) \frac{u_{1}}{u}
$$

where $u_{i}$ and $u_{i j}$ denote the corresponding partial derivatives of $u$ evaluated at $\left(c_{t}^{b g}, l\right)$. If $u\left(c_{t}, l_{t}\right)=$ $\log c_{t}+v\left(\bar{l}-l_{t}\right)$ for some function $v$, then $u$ in (63) must be interpreted to mean $\log c_{t}+v\left(\bar{l}-l_{t}\right)+$ $\frac{\log G}{1-\frac{G}{1+r}}$.

Proof: See Appendix. 
Note that (63) agrees with Proposition 2 when $G=1$. The larger is $G$, the smaller is $R^{a}$, since larger $G$ implies greater household wealth and ability to absorb shocks to asset values.

Corollary 11. Given Assumptions 1-7 and 8', relative risk aversion, evaluated along the balanced growth path, satisfies

$$
R^{c}\left(a_{t}^{b g} ; \theta_{t}^{b g}\right)=\frac{-u_{11}+\lambda_{t}^{b g} u_{12}}{u_{1}} \frac{c_{t}^{b g}}{1+w_{t}^{b g} \lambda_{t}^{b g}}+\alpha \frac{c_{t}^{b g} u_{1}}{u}
$$

and

$$
R^{c l}\left(a_{t}^{b g} ; \theta_{t}^{b g}\right)=\frac{-u_{11}+\lambda_{t}^{b g} u_{12}}{u_{1}} \frac{c_{t}^{b g}+w_{t}^{b g}(\bar{l}-l)}{1+w_{t}^{b g} \lambda_{t}^{b g}}+\alpha \frac{\left(c_{t}^{b g}+w_{t}^{b g}(\bar{l}-l)\right) u_{1}}{u} .
$$

If $u\left(c_{t}, l_{t}\right)=\log c_{t}+v\left(\bar{l}-l_{t}\right)$ for some function $v$, then $u$ in (64)-(65) must be interpreted to mean $\log c_{t}+v\left(\bar{l}-l_{t}\right)+\frac{\log G}{1-\frac{G}{1+r}}$.

Proof: See Appendix.

Thus, the expressions for relative risk aversion are essentially unchanged by balanced growth.

\section{Multiplier Preferences}

Multiplier preferences are a version of generalized recursive preferences defined by Hansen and Sargent (2001) and Strzalecki (2011). This section briefly reviews those preferences and derives the formulas for risk aversion with labor.

Households with multiplier preferences order state-contingent consumption and labor plans according to the recursive functional

$$
\widetilde{W}\left(c^{t}, l^{t}\right)=(1-\beta) u\left(c_{t}, l_{t}\right)-\beta \phi^{-1} \log E_{t} \exp \left(-\phi \widetilde{W}\left(c^{t+1}, l^{t+1}\right)\right)
$$

rather than (3), where $\beta$ is the household's discount factor and $\phi \in \mathbb{R}$. The preferences (66) can be regarded as a special case of (5), corresponding to $\rho=0$. Denote the maximized value of (66), subject to (1)-(2), by

$$
W\left(a_{t} ; \theta_{t}\right)=\max _{\left(c_{t}, l_{t}\right) \in \Gamma\left(a_{t} ; \theta_{t}\right)}(1-\beta) u\left(c_{t}, l_{t}\right)-\beta \phi^{-1} \log E_{t} \exp \left(-\phi W\left(a_{t+1} ; \theta_{t+1}\right)\right) .
$$

Hansen and Sargent (2001) show how (66)-(67) can be derived from microfoundations based on household optimization in the presence of concerns regarding model misspecification. ${ }^{39}$ Maximizing (67) instead of expected utility ensures that the household achieves a reasonable discounted sum of utility flows for a range of empirically plausible processes for $\theta_{t}$.

\footnotetext{
${ }^{39}$ These microfoundations can be used to derive values of $\phi \geq 0$. The case $\phi<0$, corresponding to risk-loving behavior, cannot be microfounded this way.
} 
As $\phi$ approaches 0, (67) converges to expected utility. For $\phi \neq 0$, the intertemporal elasticity of substitution is the same as for expected utility, but the household's risk aversion can be amplified (or attenuated) by the additional curvature parameter $\phi$.

From a practical perspective, an advantage of multiplier preferences is that they are welldefined even when $u$ takes on positive and negative values, so Assumption 2 can be dropped. Modifying the other assumptions and definitions to correspond to $W$ rather than $V$ gives the following:

Proposition 12. Let $\left(a_{t} ; \theta_{t}\right)$ be an interior point of $X$. Given Assumptions 1 and $3-6, \hat{W}\left(a_{t} ; \theta_{t} ; \sigma\right)$, $\mu\left(a_{t} ; \theta_{t} ; \sigma\right)$, and $R^{a}\left(a_{t} ; \theta_{t}\right)$ exist, and

$$
R^{a}\left(a_{t} ; \theta_{t}\right)=\frac{-E_{t} \exp \left(-\phi W\left(a_{t+1}^{*} ; \theta_{t+1}\right)\right)\left[W_{11}\left(a_{t+1}^{*} ; \theta_{t+1}\right)-\phi W_{1}\left(a_{t+1}^{*} ; \theta_{t+1}\right)^{2}\right]}{E_{t} \exp \left(-\phi W\left(a_{t+1}^{*} ; \theta_{t+1}\right)\right) W_{1}\left(a_{t+1}^{*} ; \theta_{t+1}\right)}
$$

Given Assumptions 7-8, (68) can be evaluated at the steady state to yield:

$$
R^{a}(a ; \theta)=\frac{-W_{11}(a ; \theta)}{W_{1}(a ; \theta)}+\phi W_{1}(a ; \theta) .
$$

Proof: The proof follows along exactly the same lines as Proposition 1.

Even though the preferences (67) can be derived from a concern for robustness rather than risk, the household acts in a way that is observationally equivalent to having higher risk aversion. That is, if one confronts a Hansen-Sargent household with the hypothetical gamble in (7), the household's concerns about the stochastic process $\left\{\theta_{t}\right\}$ manifest themselves as an increased aversion to the gamble; as a result, the household behaves exactly as if it were certain about the economic environment but had a higher level of risk aversion governed by $\phi$. Higher values of $\phi$ correspond to higher levels of risk aversion, with sufficiently negative values of $\phi$ corresponding to risk-loving behavior.

Proposition 13. Given Assumptions 1 and 3-8, the household's coefficient of absolute risk aversion, $R^{a}\left(a_{t} ; \theta_{t}\right)$, in (69) satisfies

$$
R^{a}(a ; \theta)=\frac{-u_{11}+\lambda u_{12}}{u_{1}} \frac{r}{1+w \lambda}+\phi r u_{1} .
$$

Proof: The proof follows along the same lines as Proposition 2.

Corollary 14. Given Assumptions 1 and 3-8, relative risk aversion for multiplier preferences, evaluated at steady state, satisfies

$$
R^{c}(a ; \theta)=\frac{-u_{11}+\lambda u_{12}}{u_{1}} \frac{c}{1+w \lambda}+\phi c u_{1}
$$


and

$$
R^{c l}(a ; \theta)=\frac{-u_{11}+\lambda u_{12}}{u_{1}} \frac{c+w(\bar{l}-l)}{1+w \lambda}+\phi(c+w(\bar{l}-l)) u_{1} .
$$

A noteworthy feature of multiplier preferences is that additive shifts of the period utility function $u$ have no effect on risk aversion, while multiplicative scalings of $u$ do affect risk aversion. (For standard Epstein-Zin-Weil preferences, it is the other way around.) In particular, the expressions (70)-(72) only hold when the period utility function $u\left(c_{t}, l_{t}\right)$ is premultiplied by $(1-\beta)$, as in (66) and (67); without that scaling factor, the second terms of (70)-(72) would each need to be multiplied by $(1-\beta)^{-1}=\frac{1+r}{r}$. If $\beta \approx .99$, this would be observationally equivalent to increasing $\phi$ by a factor of about 100, a large increase in risk aversion for what might seem like a simple renormalization of the preference specification.

Example 6. Tallarini (2000) considers the multiplier specification (67) with period utility

$$
u\left(c_{t}, l_{t}\right)=\frac{1}{1+\xi} \log c_{t}+\frac{\xi}{1+\xi} \log \left(\bar{l}-l_{t}\right),
$$

where $\xi \geq 0$. The household's consumption-wealth coefficient of relative risk aversion is given by

$$
R^{c}(a ; \theta)=\frac{-u_{11}+\lambda u_{12}}{u_{1}} \frac{c}{1+w \lambda}+\phi c u_{1}=\frac{1+\phi}{1+\xi}
$$

while including the value of leisure in household wealth gives

$$
R^{c l}(a ; \theta)=\frac{-u_{11}+\lambda u_{12}}{u_{1}} \frac{c+w(\bar{l}-l)}{1+w \lambda}+\phi(c+w(\bar{l}-l)) u_{1}=1+\phi .
$$

Neither of these equals the traditional, fixed-labor measure of risk aversion reported by Tallarini,

$$
R^{f l}(a ; \theta)=\frac{-c u_{11}}{u_{1}}+\phi c u_{1}=1+\frac{\phi}{1+\xi}
$$

This last measure ignores the fact that households will vary their labor endogenously in response to shocks. Note that $R^{c} \leq R^{f l}$, as always, although in this particular example the difference is not very large quantitatively.

\section{Discussion and Conclusions}

Traditional studies of risk aversion, such as Arrow (1964), Pratt (1965), Epstein and Zin (1989), and Weil (1989), assume that household labor supply is fixed. In standard macroeconomic models, this assumption ignores households' ability to partially offset shocks to asset values by varying hours of work. As a result, these fixed-labor measures of risk aversion are not representative of 
households' aversion to holding risky assets when labor supply can vary. For reasonable parameterizations, traditional, fixed-labor measures of risk aversion can overstate the household's actual aversion to risk by a factor of as much as ten, as in Figure 3. Fixed-labor measures of risk aversion are also unrelated to the equity premium in a standard RBC model, while the flexible-labor measure $R^{c}$ derived in the present paper is much more closely related.

Applying the Epstein-Zin-Weil fixed-labor measure of risk aversion to a Cobb-Douglas aggregate of consumption and leisure, as is sometimes done in the literature, is also problematic. If labor and consumption appear separately elsewhere in the model, such as in the production function, then consumption and leisure do not form a true composite good in the model. As a result, a composite-good measure of risk aversion is not necessarily appropriate, and in fact, turns out to be poorly correlated with the equity premium. In contrast, the consumption-wealth coefficient of relative risk aversion $R^{c}$ defined in the present paper is more closely related. This measure recognizes the household's ability to partially offset portfolio shocks by varying hours of work, but - unlike the Cobb-Douglas aggregate - excludes the value of leisure from household wealth.

The flexible-labor risk aversion measure $R^{c}$ is less than both the traditional, fixed-labor measure, $R^{f l}$, and the Cobb-Douglas aggregate measure, $R^{c l}$, described above. As a result, many studies in the macroeconomics, macro-finance, and international finance literatures may be overstating the relevant degree of risk aversion in their models.

For multiplier preferences, risk aversion is sensitive to multiplicative transformations of the period utility function $u$. Ignoring a scale factor of $1-\beta$ in period utility can lead to estimates of risk aversion that are off by a factor of 100. Thus, care must be taken to account correctly for any scale factor in utility when computing risk aversion for households with multiplier preferences.

The closed-form expressions for risk aversion derived in the present paper, and the methods of the paper more generally, are potentially useful for asset pricing in any dynamic model with multiple goods in the utility function. Models with home production, money in the utility function, or tradeable and nontradeable goods can imply very different household attitudes toward risk than traditional measures of risk aversion might suggest. 


\section{Appendix: Proofs of Propositions and Numerical Solution Details}

\section{Proof of Proposition 1}

Since $\left(a_{t} ; \theta_{t}\right)$ is an interior point of $X, V\left(a_{t}+\frac{\sigma \underline{\varepsilon}}{1+r_{t}} ; \theta_{t}\right)$ and $V\left(a_{t}+\frac{\sigma \bar{\varepsilon}}{1+r_{t}} ; \theta_{t}\right)$ exist for sufficiently small $\sigma$, and $V\left(a_{t}+\frac{\sigma \varepsilon}{1+r_{t}} ; \theta_{t}\right) \leq \hat{V}\left(a_{t} ; \theta_{t} ; \sigma\right) \leq V\left(a_{t}+\frac{\sigma \bar{\varepsilon}}{1+r_{t}} ; \theta_{t}\right)$, hence $\hat{V}\left(a_{t} ; \theta_{t} ; \sigma\right)$ exists. Moreover, since $V(\cdot ; \cdot)$ is continuous and increasing in its first argument, the intermediate value theorem implies there exists a unique $-\mu(\sigma) \in[\sigma \underline{\varepsilon}, \sigma \bar{\varepsilon}]$ with $V\left(a_{t}-\frac{\mu(\sigma)}{1+r_{t}} ; \theta_{t}\right)=\hat{V}\left(a_{t} ; \theta_{t} ; \sigma\right)$.

For generalized recursive preferences, the household's first-order optimality conditions for $c_{t}^{*}$ and $l_{t}^{*}$,

$$
\begin{aligned}
& u_{1}\left(c_{t}^{*}, l_{t}^{*}\right)=\beta\left(E_{t} V\left(a_{t+1}^{*} ; \theta_{t+1}\right)^{1-\alpha}\right)^{\alpha /(1-\alpha)} E_{t} V\left(a_{t+1}^{*} ; \theta_{t+1}\right)^{-\alpha} V_{1}\left(a_{t+1}^{*} ; \theta_{t+1}\right), \\
& u_{2}\left(c_{t}^{*}, l_{t}^{*}\right)=-\beta w_{t}\left(E_{t} V\left(a_{t+1}^{*} ; \theta_{t+1}\right)^{1-\alpha}\right)^{\alpha /(1-\alpha)} E_{t} V\left(a_{t+1}^{*} ; \theta_{t+1}\right)^{-\alpha} V_{1}\left(a_{t+1}^{*} ; \theta_{t+1}\right),
\end{aligned}
$$

are slightly more complicated than the case of expected utility considered in Swanson (2012). Note that (A1) and (A2) are related by the usual $u_{2}\left(c_{t}^{*}, l_{t}^{*}\right)=-w_{t} u_{1}\left(c_{t}^{*} ; l_{t}^{*}\right)$, and when $\alpha=0,(\mathrm{~A} 1)$ and (A2) reduce to the standard optimality conditions for expected utility.

For an infinitesimal fee $d \mu$ in (8), the first-order change in household welfare (4) is given by

$$
-V_{1}\left(a_{t} ; \theta_{t}\right) \frac{d \mu}{1+r_{t}}
$$

Differentiating (6) with respect to $a_{t}$ yields

$$
\begin{aligned}
V_{1}\left(a_{t} ; \theta_{t}\right)= & u_{1}\left(c_{t}^{*}, l_{t}^{*}\right) \frac{\partial c_{t}^{*}}{\partial a_{t}}+u_{2}\left(c_{t}^{*}, l_{t}^{*}\right) \frac{\partial l_{t}^{*}}{\partial a_{t}} \\
& +\beta\left(E_{t} V\left(a_{t+1}^{*} ; \theta_{t+1}\right)^{1-\alpha}\right)^{\alpha /(1-\alpha)} E_{t} V\left(a_{t+1}^{*} ; \theta_{t+1}\right)^{-\alpha} V_{1}\left(a_{t+1}^{*} ; \theta_{t+1}\right)\left[\left(1+r_{t}\right)-\frac{\partial c_{t}^{*}}{\partial a_{t}}+w_{t} \frac{\partial l_{t}^{*}}{\partial a_{t}}\right] .
\end{aligned}
$$

Applying (A1)-(A2) to (A4) gives the envelope theorem,

$$
V_{1}\left(a_{t} ; \theta_{t}\right)=\beta\left(1+r_{t}\right)\left(E_{t} V\left(a_{t+1}^{*} ; \theta_{t+1}\right)^{1-\alpha}\right)^{\alpha /(1-\alpha)} E_{t} V\left(a_{t+1}^{*} ; \theta_{t+1}\right)^{-\alpha} V_{1}\left(a_{t+1}^{*} ; \theta_{t+1}\right)
$$

and the Benveniste-Scheinkman equation (11),

$$
V_{1}\left(a_{t} ; \theta_{t}\right)=\left(1+r_{t}\right) u_{1}\left(c_{t}^{*}, l_{t}^{*}\right) .
$$

From (A5), (A3) equals

$$
-\beta\left(E_{t} V\left(a_{t+1}^{*} ; \theta_{t+1}\right)^{1-\alpha}\right)^{\alpha /(1-\alpha)} E_{t} V\left(a_{t+1}^{*} ; \theta_{t+1}\right)^{-\alpha} V_{1}\left(a_{t+1}^{*} ; \theta_{t+1}\right) d \mu .
$$

Turning now to the gamble in (7), the household's optimal choices for consumption and labor in period $t, c_{t}^{*}$ and $l_{t}^{*}$, will generally depend on the size of the gamble $\sigma$-for example, the household may undertake precautionary saving when faced with this gamble. Thus, in this section we write $c_{t}^{*} \equiv$ $c^{*}\left(a_{t} ; \theta_{t} ; \sigma\right)$ and $l_{t}^{*} \equiv l^{*}\left(a_{t} ; \theta_{t} ; \sigma\right)$ to emphasize this dependence on $\sigma$. The household's value function, inclusive of the one-shot gamble in $(7)$, satisfies

$$
\hat{V}\left(a_{t} ; \theta_{t} ; \sigma\right)=u\left(c_{t}^{*}, l_{t}^{*}\right)+\beta E_{t} V\left(a_{t+1}^{*} ; \theta_{t+1}\right),
$$

where $a_{t+1}^{*} \equiv\left(1+r_{t}\right) a_{t}+w_{t} l_{t}^{*}+d_{t}-c_{t}^{*}$. Because (7) describes a one-shot gamble in period $t$, it affects assets $a_{t+1}^{*}$ in period $t+1$ but otherwise does not affect the household's optimization problem from period $t+1$ onward; as a result, the household's value-to-go at time $t+1$ is just $V\left(a_{t+1}^{*} ; \theta_{t+1}\right)$, which does not depend on $\sigma$ except through $a_{t+1}^{*}$.

Differentiating (A8) with respect to $\sigma$, the first-order effect of the gamble on household welfare is:

$$
\left[u_{1} \frac{\partial c^{*}}{\partial \sigma}+u_{2} \frac{\partial l^{*}}{\partial \sigma}+\beta\left(E_{t} V^{1-\alpha}\right)^{\alpha /(1-\alpha)} E_{t} V^{-\alpha} V_{1} \cdot\left(w_{t} \frac{\partial l^{*}}{\partial \sigma}-\frac{\partial c^{*}}{\partial \sigma}+\varepsilon_{t+1}\right)\right] d \sigma
$$


where the arguments of $u_{1}, u_{2}, V$, and $V_{1}$ are suppressed to simplify notation. Optimality of $c_{t}^{*}$ and $l_{t}^{*}$ implies that the terms involving $\partial c^{*} / \partial \sigma$ and $\partial l^{*} / \partial \sigma$ cancel, as in the usual envelope theorem (these derivatives vanish at $\sigma=0$ anyway, for the reasons discussed below). Moreover, $E_{t} V^{-\alpha} V_{1} \varepsilon_{t+1}=0$ because $\varepsilon_{t+1}$ is independent of $\theta_{t+1}$ and $a_{t+1}^{*}$, evaluating the latter at $\sigma=0$. Thus, the first-order cost of the gamble is zero, as in Arrow (1964) and Pratt (1965).

To second order, the effect of the gamble on household welfare is

$$
\begin{aligned}
\left\{u_{11}\left(\frac{\partial c^{*}}{\partial \sigma}\right)^{2}\right. & +2 u_{12} \frac{\partial c^{*}}{\partial \sigma} \frac{\partial l^{*}}{\partial \sigma}+u_{22}\left(\frac{\partial l^{*}}{\partial \sigma}\right)^{2}+u_{1} \frac{\partial^{2} c^{*}}{\partial \sigma^{2}}+u_{2} \frac{\partial^{2} l^{*}}{\partial \sigma^{2}} \\
& +\alpha \beta\left(E_{t} V^{1-\alpha}\right)^{(2 \alpha-1) /(1-\alpha)}\left[E_{t} V^{-\alpha} V_{1} \cdot\left(w_{t} \frac{\partial l^{*}}{\partial \sigma}-\frac{\partial c^{*}}{\partial \sigma}+\varepsilon_{t+1}\right)\right]^{2} \\
& -\alpha \beta\left(E_{t} V^{1-\alpha}\right)^{\alpha /(1-\alpha)} E_{t} V^{-\alpha-1}\left[V_{1} \cdot\left(w_{t} \frac{\partial l^{*}}{\partial \sigma}-\frac{\partial c^{*}}{\partial \sigma}+\varepsilon_{t+1}\right)\right]^{2} \\
& +\beta\left(E_{t} V^{1-\alpha}\right)^{\alpha /(1-\alpha)} E_{t} V^{-\alpha} V_{11} \cdot\left(w_{t} \frac{\partial l^{*}}{\partial \sigma}-\frac{\partial c^{*}}{\partial \sigma}+\varepsilon_{t+1}\right)^{2} \\
& \left.+\beta\left(E_{t} V^{1-\alpha}\right)^{\alpha /(1-\alpha)} E_{t} V^{-\alpha} V_{1} \cdot\left(w_{t} \frac{\partial^{2} l^{*}}{\partial \sigma^{2}}-\frac{\partial^{2} c^{*}}{\partial \sigma^{2}}\right)\right\} \frac{d \sigma^{2}}{2} .
\end{aligned}
$$

The terms involving $\partial^{2} c^{*} / \partial \sigma^{2}$ and $\partial^{2} l^{*} / \partial \sigma^{2}$ cancel due to the optimality of $c_{t}^{*}$ and $l_{t}^{*}$. The derivatives $\partial c^{*} / \partial \sigma$ and $\partial l^{*} / \partial \sigma$ vanish at $\sigma=0$ (there are two ways to see this: first, the linearized version of the model is certainty equivalent; alternatively, if the distribution of $\varepsilon$ is symmetric about zero, the gamble in (7) is isomorphic for positive and negative $\sigma$, hence $c^{*}$ and $l^{*}$ must be symmetric about $\sigma=0$, implying the derivatives vanish). Finally, $\varepsilon_{t+1}$ is independent of $\theta_{t+1}$ and $a_{t+1}^{*}$, evaluating the latter at $\sigma=0$. Since $\varepsilon_{t+1}$ has unit variance, (A10) reduces to

$$
\beta\left(E_{t} V^{1-\alpha}\right)^{\alpha /(1-\alpha)}\left(E_{t} V^{-\alpha} V_{11}-\alpha E_{t} V^{-\alpha-1} V_{1}^{2}\right) \frac{d \sigma^{2}}{2} .
$$

Equating (A7) to (A11) allows us to solve for $d \mu$ as a function of $d \sigma^{2}$. Thus, $\lim _{\sigma \rightarrow 0} 2 \mu(\sigma) / \sigma^{2}$ exists and is given by

$$
\frac{-E_{t} V^{-\alpha} V_{11}+\alpha E_{t} V^{-\alpha-1} V_{1}^{2}}{E_{t} V^{-\alpha} V_{1}}
$$

Since (A12) is already evaluated at $\sigma=0$, to evaluate it at the nonstochastic steady state, set $a_{t+1}=a$ and $\theta_{t+1}=\theta$ to get

$$
\frac{-V_{11}(a ; \theta)}{V_{1}(a ; \theta)}+\alpha \frac{V_{1}(a ; \theta)}{V(a ; \theta)}
$$

\section{Proof of Lemma 2}

Equations (A1), (A4), and the envelope theorem imply the household's intertemporal optimality (Euler) condition,

$$
u_{1}\left(c_{t}^{*}, l_{t}^{*}\right)=\beta\left(E_{t} V\left(a_{t+1}^{*} ; \theta_{t+1}\right)^{1-\alpha}\right)^{\alpha /(1-\alpha)} E_{t} V\left(a_{t+1}^{*} ; \theta_{t+1}\right)^{-\alpha}\left(1+r_{t+1}\right) u_{1}\left(c_{t+1}^{*}, l_{t+1}^{*}\right) .
$$

Differentiating (A14) with respect to $a_{t}$ at the nonstochastic steady state implies

$$
u_{11}\left(\frac{\partial c_{t}^{*}}{\partial a_{t}}-E_{t} \frac{\partial c_{t+1}^{*}}{\partial a_{t}}\right)=-u_{12}\left(\frac{\partial l_{t}^{*}}{\partial a_{t}}-E_{t} \frac{\partial l_{t+1}^{*}}{\partial a_{t}}\right)
$$

in a neighborhood of the steady state, where the arguments of the $u_{i j}$ are suppressed to reduce notation. Using (14), this implies

$$
\left(u_{11}-\lambda u_{12}\right)\left(\frac{\partial c_{t}^{*}}{\partial a_{t}}-E_{t} \frac{\partial c_{t+1}^{*}}{\partial a_{t}}\right)=0
$$


and thus

$$
E_{t} \frac{\partial c_{t+1}^{*}}{\partial a_{t}}=\frac{\partial c_{t}^{*}}{\partial a_{t}}
$$

Equations (A14)-(A17) can be iterated forward to yield

$$
E_{t} \frac{\partial c_{t+k}^{*}}{\partial a_{t}}=\frac{\partial c_{t}^{*}}{\partial a_{t}}, \quad k=1,2, \ldots
$$

whatever the initial response $\partial c_{t}^{*} / \partial a_{t}$. From (14) and (A18), it also follows that

$$
E_{t} \frac{\partial l_{t+k}^{*}}{\partial a_{t}}=\frac{\partial l_{t}^{*}}{\partial a_{t}}, \quad k=1,2, \ldots
$$

It remains to solve for $\partial c_{t}^{*} / \partial a_{t}$. The household's intertemporal budget constraint, evaluated at steady state, implies

$$
\frac{1+r}{r} \frac{\partial c_{t}^{*}}{\partial a_{t}}=(1+r)+w \frac{1+r}{r} \frac{\partial l_{t}^{*}}{\partial a_{t}} .
$$

Substituting (14) into (A20) and solving for $\partial c_{t}^{*} / \partial a_{t}$ yields

$$
\frac{\partial c_{t}^{*}}{\partial a_{t}}=\frac{r}{1+w \lambda}
$$

\section{Proof of Lemma 5}

Differentiating the household's Euler equation (A14) at the nonstochastic steady state implies

$$
u_{11}\left(d c_{t}^{*}-E_{t} d c_{t+1}^{*}\right)+u_{12}\left(d l_{t}^{*}-E_{t} d l_{t+1}^{*}\right)=\beta u_{1} E_{t} d r_{t+1},
$$

which, applying (46), becomes

$$
\left(u_{11}-\lambda u_{12}\right)\left(d c_{t}^{*}-E_{t} d c_{t+1}^{*}\right)-\frac{u_{1} u_{12}}{u_{22}+w u_{12}}\left(d w_{t}-E_{t} d w_{t+1}\right)=\beta u_{1} E_{t} d r_{t+1} .
$$

Note that (A23) implies, for each $k=1,2, \ldots$,

$$
E_{t} d c_{t+k}^{*}=d c_{t}^{*}-\frac{u_{1} u_{12}}{u_{11} u_{22}-u_{12}^{2}}\left(d w_{t}-E_{t} d w_{t+k}\right)-\frac{\beta u_{1}}{u_{11}-\lambda u_{12}} E_{t} \sum_{i=1}^{k} d r_{t+i}
$$

Combining (1)-(2), differentiating, and evaluating at the nonstochastic steady state yields

$$
E_{t} \sum_{k=0}^{\infty} \frac{1}{(1+r)^{k}}\left(d c_{t+k}^{*}-w d l_{t+k}^{*}-l d w_{t+k}-d d_{t+k}-a d r_{t+k}\right)=(1+r) d a_{t} .
$$

Substituting (46) and (A24) into (A25), and solving for $d c_{t}^{*}$, yields

$$
\begin{aligned}
d c_{t}^{*}= & \frac{r}{1+r} \frac{1}{1+w \lambda}\left[(1+r) d a_{t}+E_{t} \sum_{k=0}^{\infty} \frac{1}{(1+r)^{k}}\left(l d w_{t+k}+d d_{t+k}+a d r_{t+k}\right)\right] \\
& +\frac{u_{1} u_{12}}{u_{11} u_{22}-u_{12}^{2}} d w_{t}+\frac{1}{1+r} \frac{-u_{1}}{u_{11}-\lambda u_{12}} E_{t} \sum_{k=0}^{\infty} \frac{1}{(1+r)^{k}}\left[\frac{r \lambda}{1+w \lambda} d w_{t+k}-\beta d r_{t+k+1}\right] .
\end{aligned}
$$

\section{Proof of Lemma 6}

Differentiating equation (6) and evaluating at the nonstochastic steady state implies

$$
d V_{t}=u_{1} d c_{t}^{*}+u_{2} d l_{t}^{*}+\beta E_{t} d V_{t+1}
$$


Solving (A27) forward and applying (46) yields

$$
d V_{t}=\sum_{k=0}^{\infty} \beta^{k} u_{1}(1+w \lambda) E_{t} d c_{t+k}^{*}-\sum_{k=0}^{\infty} \beta^{k} \frac{u_{1} u_{2}}{u_{22}+w u_{12}} E_{t} d w_{t+k} .
$$

Substituting (A24) into (A28) and simplifying yields

$$
\begin{aligned}
d V_{t}=\frac{1+r}{r} & u_{1}(1+w \lambda) d c_{t}^{*}-\frac{1+r}{r} \frac{u_{1}^{2} u_{12}(1+w \lambda)}{u_{11} u_{22}-u_{12}^{2}} d w_{t} \\
& +\sum_{k=0}^{\infty} \beta^{k} \frac{u_{1}\left(u_{1} u_{12}-u_{2} u_{11}\right)}{u_{11} u_{22}-u_{12}^{2}} E_{t} d w_{t+k}-\frac{u_{1}^{2}(1+w \lambda)}{u_{11}-\lambda u_{12}} \frac{1}{1-\beta} \sum_{k=1}^{\infty} \beta^{k+1} E_{t} d r_{t+k} .
\end{aligned}
$$

Substituting (A26) into (A29) and simplifying gives

$$
d V_{t}=u_{1}(1+r) d a_{t}+u_{1} E_{t} \sum_{k=0}^{\infty} \frac{1}{(1+r)^{k}}\left(l d w_{t+k}+d d_{t+k}+a d r_{t+k}\right) .
$$

\section{Proof of Lemma 9}

i) The household's Euler equation (A14), evaluated along the (nonstochastic) balanced growth path, implies

$$
u_{1}\left(c_{t}^{b g}, l\right)=\beta(1+r) u_{1}\left(c_{t+1}^{b g}, l\right)=\beta(1+r) u_{1}\left(G c_{t}^{b g}, l\right) .
$$

Similarly, for labor,

$$
u_{2}\left(c_{t}^{b g}, l\right)=\beta(1+r) \frac{w_{t}^{b g}}{w_{t+1}^{b g}} u_{2}\left(c_{t+1}^{b g}, l\right)=\beta(1+r) G^{-1} u_{2}\left(G c_{t}^{b g}, l\right) .
$$

As in King, Plosser, and Rebelo (2002), assume that preferences $u$ are consistent with balanced growth for all initial asset stocks and wages in a neighborhood of $a_{t}^{b g}$ and $w_{t}^{b g}$, and hence for all initial values of $\left(c_{t}, l_{t}\right)$ in a neighborhood of $\left(c_{t}^{b g}, l\right)$. Thus, (A31) and (A32) can be differentiated to yield:

$$
\begin{aligned}
& u_{11}\left(c_{t}^{b g}, l\right)=\beta(1+r) G u_{11}\left(G c_{t}^{b g}, l\right), \\
& u_{12}\left(c_{t}^{b g}, l\right)=\beta(1+r) u_{12}\left(G c_{t}^{b g}, l\right), \\
& u_{22}\left(c_{t}^{b g}, l\right)=\beta(1+r) G^{-1} u_{22}\left(G c_{t}^{b g}, l\right) .
\end{aligned}
$$

Substituting (A33)-(A35) into (15) gives

$$
\lambda_{t+1}^{b g}=\frac{w_{t+1}^{b g} u_{11}\left(c_{t+1}^{b g}, l\right)+u_{12}\left(c_{t+1}^{b g}, l\right)}{u_{22}\left(c_{t+1}^{b g}, l\right)+w_{t+1}^{b g} u_{12}\left(c_{t+1}^{b g}, l\right)}=G^{-1} \lambda_{t}^{b g},
$$

ii) Assumptions 1-6 imply (11)-(15) in the text and the Euler equation (A14). Hence

$$
\left(u_{11}\left(c_{t}^{b g}, l\right)-\lambda_{t}^{b g} u_{12}\left(c_{t}^{b g}, l\right)\right) \frac{\partial c_{t}^{*}}{\partial a_{t}}=\beta(1+r)\left(u_{11}\left(c_{t+1}^{b g}, l\right)-\lambda_{t+1}^{b g} u_{12}\left(c_{t+1}^{b g}, l\right)\right) \frac{\partial c_{t+1}^{*}}{\partial a_{t}} .
$$

Solving for $\partial c_{t+1}^{*} / \partial a_{t}$ and using (A33)-(A36) yields $\partial c_{t+1}^{*} / \partial a_{t}=G \partial c_{t}^{*} / \partial a_{t}$.

iii) Follows from (14), (A33)-(A36), and ii).

iv) Use the household's budget constraint (1)-(2) and ii) to solve for $\partial c_{t}^{*} / \partial a_{t}$.

\section{Proof of Proposition 10}

Proposition 1 implies (62). Assumptions 1-6 imply (11)-(15). Substituting (11)-(14) and Lemma 9(iv) into (62) gives

$$
R^{a}\left(a_{t}^{b g} ; \theta_{t}^{b g}\right)=\frac{-u_{11}\left(c_{t+1}^{b g}, l\right)+\lambda_{t+1}^{b g} u_{12}\left(c_{t+1}^{b g}, l\right)}{u_{1}\left(c_{t+1}^{b g}, l\right)} \frac{1+r-G}{1+w_{t+1}^{b g} \lambda_{t+1}^{b g}}+\alpha \frac{(1+r) u_{1}\left(c_{t+1}^{b g}, l\right)}{V\left(a_{t+1}^{b g} ; \theta_{t+1}^{b g}\right)}
$$


Expressing $V\left(a_{t+1}^{b g} ; \theta_{t+1}^{b g}\right)$ in terms of period utility $u$ is made slightly more complicated by the presence of balanced growth, since now the arguments of $u$ are not constant but rather grow over time.

King, Plosser, and Rebelo $(1988,2002)$ show that, to be consistent with balanced growth, $u\left(c_{t}, l_{t}\right)$ must have the functional form

$$
u\left(c_{t}, l_{t}\right)=\frac{c_{t}^{1-\gamma}}{1-\gamma} v\left(\bar{l}-l_{t}\right)
$$

or, as $\gamma \rightarrow 1$,

$$
u\left(c_{t}, l_{t}\right)=\log c_{t}+v\left(\bar{l}-l_{t}\right),
$$

where $v(\cdot)$ in (A39) or (A40) is differentiable, increasing, and concave, but otherwise unrestricted. Since the balanced growth path is nonstochastic, the allowable functional forms for $u\left(c_{t}, l_{t}\right)$ are the same for the case of generalized recursive preferences as they are for expected utility.

If $u$ has the form (A39), then

$$
V\left(a_{t}^{b g} ; \theta_{t}^{b g}\right)=\frac{1}{1-\beta G^{1-\gamma}} u\left(c_{t}^{b g}, l\right)
$$

and

$$
\beta V\left(a_{t+1}^{b g} ; \theta_{t+1}^{b g}\right)=V\left(a_{t}^{b g} ; \theta_{t}^{b g}\right)-u\left(c_{t}^{b g}, l\right)=\frac{\beta G^{1-\gamma}}{1-\beta G^{1-\gamma}} u\left(c_{t}^{b g}, l\right) .
$$

Moreover, $\beta(1+r)=G^{\gamma}$. Substituting (A31), (A33)-(A35), and (A42) into (A38) then completes the proof.

If $u$ has the form (A40), then

$$
\begin{gathered}
V\left(a_{t}^{b g} ; \theta_{t}^{b g}\right)=\frac{1}{1-\beta} u\left(c_{t}^{b g}, l\right)+\frac{\beta}{(1-\beta)^{2}} \log G, \\
\beta V\left(a_{t+1}^{b g} ; \theta_{t+1}^{b g}\right)=\frac{\beta}{1-\beta} u\left(c_{t}^{b g}, l\right)+\frac{\beta}{(1-\beta)^{2}} \log G,
\end{gathered}
$$

and $\beta(1+r)=G$. Substituting (A31), (A33)-(A36), and (A44) into (A38) yields

$$
R^{a}\left(a_{t}^{b g} ; \theta_{t}^{b g}\right)=\frac{-u_{11}+\lambda_{t}^{b g} u_{12}}{u_{1}} \frac{\frac{1+r}{G}-1}{1+w_{t}^{b g} \lambda_{t}^{b g}}+\alpha\left(\frac{1+r}{G}-1\right) \frac{u_{1}}{u+\frac{1+r}{1+r-G} \log G} .
$$

This differs from (63) by the addition of the constant term $\frac{\log G}{1-\frac{G}{1+r}}$ to $u$. Thus, in the case of log preferences, $u$ in (63) must be interpreted to include the additive constant $\frac{\log G}{1-\frac{G}{1+r}}$.

\section{Proof of Corollary 11}

As in Definitions 2-3, define wealth $A_{t}^{b g}$ in beginning- rather than end-of-period- $t$ units; this requires multiplying by $(1+r)^{-1} G$ rather than just $(1+r)^{-1}$. Then the present discounted value of consumption along the balanced growth path is given by $A_{t}^{b g}=c_{t}^{b g} /\left(\frac{1+r}{G}-1\right)$, and the present discounted value of leisure by $w_{t}^{b g}(\bar{l}-l) /\left(\frac{1+r}{G}-1\right)$. Substituting these into Proposition 10 completes the proof.

\section{Numerical Solution Procedure for Sections 4-5}

The equations of the RBC model itself are standard:

$$
\begin{gathered}
Y_{t}=Z_{t} K_{t-1}^{1-\theta} L_{t}^{\theta}, \\
K_{t}=(1-\delta) K_{t-1}+Y_{t}-C_{t}, \\
\eta L_{t}^{\chi} / C_{t}^{-\gamma}=w_{t}, \\
r_{t}=(1-\theta) Y_{t} / K_{t-1}-\delta, \\
w_{t}=\theta Y_{t} / L_{t},
\end{gathered}
$$




$$
\log Z_{t}=\rho \log Z_{t-1}+\varepsilon_{t}
$$

where, for concreteness, the additively separable preference specification from Examples 3 and 5 have been used in (A49), and will be assumed throughout this section. In equations (A46)-(A51), note that $K_{t-1}$ denotes the capital stock at the beginning of period $t$ (or the end of period $t-1$ ), so the notation differs slightly from the main text for compatibility with the numerical algorithm below.

Because of the generalized recursive structure of household preferences, the household's Euler equation (A14) involves the value function. Following Rudebusch and Swanson (2012), two equations for the value function are added to the model, as follows:

$$
\begin{gathered}
V_{t}=\frac{C_{t}^{1-\gamma}}{1-\gamma}-\eta \frac{L_{t}^{1+\chi}}{1+\chi}+\beta \operatorname{VTWIST}_{t}^{1 /(1-\alpha)}, \\
\operatorname{VTWIST}_{t}=E_{t} V_{t+1}^{1-\alpha} .
\end{gathered}
$$

The household's Euler equation (A14) then can be written as

$$
C_{t}^{-\gamma}=\beta E_{t}\left(1+r_{t+1}\right)\left(V_{t+1} / \operatorname{VTWIST}_{t}^{1 /(1-\alpha)}\right)^{-\alpha} C_{t+1}^{-\gamma}
$$

To compute risk aversion, the following auxiliary variables and equations must be appended to (A46)-(A54):

$$
\begin{gathered}
\lambda_{t}=(\gamma / \chi) L_{t} / C_{t}, \\
C_{t}^{-\gamma-1} \mathrm{DCDA}_{t}=\beta E_{t}\left(1+r_{t+1}\right) C_{t+1}^{-\gamma-1} \mathrm{DCDA}_{t+1}\left[\left(1+r_{t}\right)-\left(1+w_{t} \lambda_{t}\right) \mathrm{DCDA}_{t}\right] \\
\mathrm{CARA}_{t}=\frac{E_{t} V_{t+1}^{-\alpha}\left[\left(1+r_{t+1}\right)\left(\gamma C_{t+1}^{-\gamma-1} \mathrm{DCDA}_{t+1}\right)+\alpha\left(1+r_{t+1}\right)^{2} C_{t+1}^{-2 \gamma} / V_{t+1}\right]}{\mathrm{V} 1 \mathrm{EXP}_{t}} \\
\mathrm{~V} 1 \mathrm{EXP}_{t}=E_{t} V_{t+1}^{-\alpha}\left(1+r_{t+1}\right) C_{t+1}^{-\gamma} . \\
\mathrm{PDVC}_{t}=C_{t}+\beta E_{t} C_{t+1}^{-\gamma} / C_{t}^{-\gamma}\left(V_{t+1} / \mathrm{VTWIST}_{t}^{1 /(1-\alpha)}\right)^{-\alpha} \mathrm{PDVC}_{t+1} . \\
\mathrm{CRRA}_{t}=\mathrm{CARA}_{t} \mathrm{PDVC}_{t} /\left(1+r_{t}\right) .
\end{gathered}
$$

These are somewhat more complicated versions of the equations in Swanson (2012), owing to the use of generalized recursive preferences in the present paper. Equation (A55) corresponds to (14), (A57)-(A58) to Proposition 1, and (A59)-(A60) to Definition 2. The variable $\mathrm{DCDA}_{t}$ correspond to $\partial c_{t}^{*} / \partial a_{t}$, and equation (A56) is the derivative of (A14) with respect to $a_{t}$, which is what determines how $\partial c_{t}^{*} / \partial a_{t}$ evolves over time. Note that

$$
\frac{\partial c_{t+1}^{*}}{\partial a_{t}}=\frac{\partial c_{t+1}^{*}}{\partial a_{t+1}^{*}}\left[\left(1+r_{t}\right)-w_{t} \lambda_{t} \frac{\partial c_{t}^{*}}{\partial a_{t}}-\frac{\partial c_{t}^{*}}{\partial a_{t}}\right]
$$

which is used in (A56). The envelope condition $V_{1}\left(a_{t} ; \theta_{t}\right)=\beta\left(1+r_{t}\right) E_{t} V_{1}\left(a_{t+1} ; \theta_{t+1}\right)$ is used to rewrite $E_{t} V_{1}\left(a_{t+1} ; \theta_{t+1}\right)$ in (A57)-(A58), and equations (11)-(12) are used to rewrite $V_{1}$ and $V_{11}$ in terms of derivatives of $u$.

The system of equations (A46)-(A60) can then be solved numerically using the Perturbation AIM algorithm of Swanson, Anderson, and Levin (2006) to compute a fifth-order Taylor series approximate solution around the nonstochastic steady state. These $n$ th-order Taylor series approximations are guaranteed to be arbitrarily accurate in a neighborhood of the nonstochastic steady state, but importantly also converge globally within the domain of convergence of the Taylor series as the order of the approximation $n$ becomes large. In practice, the solution seemed to converge globally over the range of values considered for the state variables in Figure 1-5 by about the third or fourth order, so solutions higher than the fifth order are not reported. Aruoba, Fernández-Villaverde, and Rubio-Ramírez (2006) solve a standard real business cycle model like (A46)-(A60) using a variety of numerical methods, including second- and fifth-order perturbation methods, and find that the perturbation solutions are among the most accurate methods globally, as well as being the fastest to compute. 


\section{References}

Alvarez, Fernando And Nancy Stokey (1998). "Dynamic Programming with Homogeneous Functions," Journal of Economic Theory 82, 167-189.

Andreasen, Martin (2012). "On the Effects of Rare Disasters and Uncertainty Shocks for Risk Premia in Nonlinear DSGE Models," Review of Economic Dynamics 15, 295-316.

Andreasen, Martin (2013). "An Estimated DSGE Model: Explaining Variation in Nominal Term Premia, Real Term Premia, and Inflation Risk Premia," European Economic Review, forthcoming.

Arrow, Kenneth (1965). "Aspects of the Theory of Risk-Bearing," Yrjö Jahnsson Lectures, in Essays in the Theory of Risk Bearing, ed. Arrow, Kenneth (Chicago: Markum, 1971).

Aruoba, S. Boragan, Jesús Fernández-Villaverde, and Juan Rubio-Ramírez (2006). "Comparing Solution Methods for Dynamic Equilibrium Economies," Journal of Economic Dynamics and Control 30, 2477-2508.

Backus, David, Bryan Routledge, and Stanley Zin (2008). "Asset Prices in Business Cycle Analysis," unpublished manuscript, Tepper School of Business, Carnegie Mellon University.

Boldrin, Michele, Lawrence Christiano, and Jonas Fisher (1997). "Habit Persistence and Asset Returns in an Exchange Economy," Macroeconomic Dynamics 1, 312-332.

Boldrin, Michele, Lawrence Christiano, and Jonas Fisher (2001). "Habit Persistence, Asset Returns, and the Business Cycle," American Economic Review 91, 149-166.

Campbell, John and John Cochrane (1999). "By Force of Habit: A Consumption-Based Explanation of Aggregate Stock Market Behavior," Journal of Political Economy 107, 205-251.

Coile, Courtney, and Paul Levine (2009). "The Market Crash and Mass Layoffs: How the Current Economic Crisis May Affect Retirement," NBER Working Paper 15395.

Colacito, Riccardo and Mariano Croce (2012). "International Asset Pricing with Recursive Preferences," unpublished manuscript, University of North Carolina Kenan-Flagler School of Business.

Constantinides, George (1990). "Habit Formation: A Resolution of the Equity Premium Puzzle," Journal of Political Economy 98, 519-543.

Coronado, Julia, and Maria Perozek (2003). "Wealth Effects and the Consumption of Leisure: Retirement Decisions During the Stock Market Boom of the 1990s," Federal Reserve Board Finance and Economics Discussion Series 2003-20.

Dew-Becker, Ian (2012). "A Model of Time-Varying Risk Premia with Habits and Production," unpublished manuscript, Duke University Fuqua School of Business.

Epstein, Larry and Stanley Zin (1989). "Substitution, Risk Aversion, and the Temporal Behavior of Consumption and Asset Returns: A Theoretical Framework," Econometrica 57, 937-969.

FARmer, Roger (1990). "RINCE Preferences," Quarterly Journal of Economics 105, 43-60.

Flavin, Marjorie and Shinobu Nakagawa (2008). "A Model of Housing in the Presence of Adjustment Costs: A Structural Interpretation of Habit Persistence," American Economic Review 98, 474-495.

Gourio, François (2012). "Disaster Risk and Business Cycles," American Economic Review 102, 27342766.

Gourio, François (2013). "Credit Risk and Disaster Risk," American Economic Journal: Macroeconomics $5,1-34$.

Hansen, Lars P. and Thomas Sargent (2001). "Robust Control and Model Uncertainty," American Economic Review 91, 60-66. 
Imbens, Guido, Donald Rubin, and Bruce Sacerdote (2001). "Estimating the Effect of Unearned Income on Labor Earnings, Savings, and Consumption: Evidence from a Survey of Lottery Players," American Economic Review 91, 778-794.

Kihlstrom, Richard and Leonard Mirman (1974). "Risk Aversion with Many Commodities," Journal of Economic Theory 8, 361-388.

King, Robert, Charles Plosser, and Sergio Rebelo (1988). "Production, Growth, and Business Cycles: I. The Basic Neoclassical Model," Journal of Monetary Economics 21, 195-232.

King, Robert, Charles Plosser, and Sergio Rebelo (2002). "Production, Growth, and Business Cycles: Technical Appendix," Computational Economics 20, 87-116.

Kreps, David and Evan Porteus (1978). "Temporal Resolution of Uncertainty and Dynamic Choice Theory," Econometrica 46, 185-200.

Kung, Howard (2012). "Equilibrium Growth, Inflation, and Bond Yields," unpublished manuscript, University of British Columbia.

Lettau, Martin and Harald Uhlig (2000). "Can Habit Formation Be Reconciled with Business Cycle Facts?" Review of Economic Dynamics 3, 79-99.

Marinacci, Massimo, and Luigi Montrucchio (2010). "Unique Solutions for Stochastic Recursive Utilities," Journal of Economic Theory 145, 1776-1804.

Merton, Robert (1971). "An Intertemporal Capital Asset Pricing Model," Econometrica 41, 867-887.

Palomino, Francisco (2012). "Bond Risk Premiums and Optimal Monetary Policy," Review of Economic Dynamics 15, 19-40.

Pencavel, John (1986). "Labor Supply of Men: A Survey," Handbook of Labor Economics 1, 3-102.

Pratt, John (1964). "Risk Aversion in the Small and in the Large," Econometrica 32, 122-136.

Rincón-Zapatero, Juan and Carlos Rodríguez-Palmero (2003). "Existence and Uniqueness of Solutions to the Bellman Equation in the Unbounded Case," Econometrica 71, 1519-55.

Rouwenhorst, K. Geert (1995). "Asset Pricing Implications of Equilibrium Business Cycle Models," in Frontiers of Business Cycle Research, ed. Cooley, Thomas (Princeton: Princeton University Press).

Rudebusch, Glenn, and Eric Swanson (2008). "Examining the Bond Premium Puzzle with a DSGE Model," Journal of Monetary Economics 55, 111-126.

Rudebusch, Glenn, and Eric Swanson (2009). "The Bond Premium in a DSGE Model with Long-Run Real and Nominal Risks," Federal Reserve Bank of SF Working Paper 2008-31.

Rudebusch, Glenn, and Eric Swanson (2012). "The Bond Premium in a DSGE Model with Long-Run Real and Nominal Risks," American Economic Journal: Macroeconomics 4, 105-143.

Santos, Manuel (1991). "Smoothness of the Policy Function in Discrete Time Economic Models," Econometrica 59, 1365-1382.

Stiglitz, Joseph (1969). "Behavior Towards Risk with Many Commodities," Econometrica 37, 660-667.

Stokey, Nancy, and Robert Lucas, with Edward Prescott (1989). Recursive Methods in Economic Dynamics (Chicago: University of Chicago Press).

Strzalecki, Tomasz (2011). "Axiomatic Foundations for Multiplier Preferences," Econometrica 79, $47-73$.

Swanson, Eric (2012). "Risk Aversion and the Labor Margin in Dynamic Equilibrium Models," American Economic Review, 102, 1663-1691.

Swanson, Eric, Gary Anderson, and Andrew Levin (2006). "Higher-Order Perturbation Solutions to Dynamic, Discrete-Time Rational Expectations Models," Federal Reserve Bank of San Francisco Working Paper 2006-01. 
Tallarini, Thomas (2000). "Risk-Sensitive Business Cycles," Journal of Monetary Economics 45, 507532.

Uhlig, Harald (2007). "Leisure, Growth, and Long-Run Risk," unpublished manuscript, University of Chicago.

Van Binsbergen, Jules, Jesús Fernández-Villaverde, Ralph Koijen, and Juan Rubio-Ramírez (2012). "The Term Structure of Interest Rates in a DSGE Model with Recursive Preferences," Journal of Monetary Economics 59, 634-648.

Weil, Phillipe (1989). "The Equity Premium Puzzle and the Risk-Free Rate Puzzle," Journal of Monetary Economics 24, 401-421. 